\title{
A Cardiomyocyte-Specific Wdr1 Knockout Demonstrates Essential Functional Roles for Actin Disassembly during Myocardial Growth and Maintenance in Mice
}

\author{
Baiyin Yuan, ${ }^{*}$ Ping Wan, ${ }^{*}$ Dandan Chu,${ }^{* \dagger}$ Junwei Nie, ${ }^{*}$ Yunshan Cao, ${ }^{*}$ Wen Luo, ${ }^{*}$ Shuangshuang Lu, ${ }^{*}$ Jiong Chen, ${ }^{*}$ and \\ Zhongzhou Yang*¥
}

\begin{abstract}
From the MOE Key Laboratory of Model Animal for Disease Study,* Model Animal Research Center, Nanjing Biomedical Research Institute, Nanjing University, Nanjing; the Jiangsu Key Laboratory of Neuroregeneration, ${ }^{\dagger}$ Nantong University, Nantong; and the State Key Laboratory of Genetic Engineering, ${ }^{\ddagger}$ Collaborative Innovation Center for Genetics and Development, Institute of Plant Biology, School of Life Sciences, Fudan University, Shanghai, China
\end{abstract}

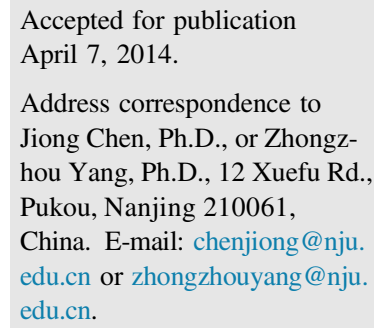

\begin{abstract}
Actin dynamics are critical for muscle development and function, and mutations leading to deregulation of actin dynamics cause various forms of heritable muscle diseases. AIP1 is a major cofactor of the actin depolymerizing factor/cofilin in eukaryotes, promoting actin depolymerizing factor/cofilin-mediated actin disassembly. Its function in vertebrate muscle has been unknown. To investigate functional roles of AIP1 in myocardium, we generated conditional knockout (cK0) mice with cardiomyocyte-specific deletion of Wdr1, the mammalian homolog of yeast AIP1. Wdr1 cK0 mice began to die at postnatal day 13 (P13), and none survived past P24. At P12, cK0 mice exhibited cardiac hypertrophy and impaired contraction of the left ventricle. Electrocardiography revealed reduced heart rate, abnormal $P$ wave, and abnormal T wave at P10 and prolonged QT interval at P12. Actin filament (F-actin) accumulations began at P10 and became prominent at P12 in the myocardium of $\mathrm{CKO}$ mice. Within regions of F-actin accumulation in myofibrils, the sarcomeric components $\alpha$-actinin and tropomodulin- 1 exhibited disrupted patterns, indicating that F-actin accumulations caused by Wdr1 deletion result in disruption of sarcomeric structure. Ectopic cofilin colocalized with F-actin aggregates. In adult mice, Wdr1 deletion resulted in similar but much milder phenotypes of heart hypertrophy, F-actin accumulations within myofibrils, and lethality. Taken together, these results demonstrate that AIP1-regulated actin dynamics play essential roles in heart function in mice. (Am J Pathol 2014, 184: 1967-1980; http://dx.doi.org/ 10.1016/j.ajpath.2014.04.007)
\end{abstract}

The dynamics of the actin cytoskeleton derive from the fact that actin filaments simultaneously undergo polymerization at their barbed (plus) ends and depolymerization at their pointed (minus) ends under physiological conditions. A large number of actin-binding proteins regulate actin assembly and disassembly. ${ }^{1}$ Among these, the Arp $2 / 3$ complex and formins are known to promote rapid polymerization of monomeric actin (G-actin) at the barbed ends, ${ }^{2,3}$ whereas actin depolymerizing factor (ADF)/cofilin is the major factor promoting severing of actin filaments (F-actin) and depolymerization of G-actin from the pointed ends. ${ }^{4}$ The dual functions of actin severing and depolymerization enable ADF/cofilin to serve as a dynamizing factor of actin cytoskeleton in cells requiring strong actin dynamics. 4
Actin-interacting protein 1 (AIP1) is a major cofactor of ADF/cofilin that actively disassembles ADF/cofilin-bound actin filaments. AIP1 can bind to the ADF/cofilin-F-actin complex and can strongly enhance the severing activity of ADF/cofilin on actin filaments by capping the barbed ends of the severed filaments and blocking their reannealing and elongation, resulting in acceleration of actin disassembly.

Supported by National Key Basic Research Program of China grants 2011 CB943904 and 2012CB966602 (Z.Y.), National Natural Science Foundation of China grants 31071282, 91019002 , and 31130037 (Z.Y.), and the Open Research Fund of State Key Laboratory of Bioelectronics, South-East University, China.

B.Y. and P.W. contributed equally to this work.

Disclosures: None declared. 
Both ADF/cofilin (17 kDa) and AIP1 (67 kDa) are highly conserved across all eukaryotes and are found to be required for a wide spectrum of actin-based processes in nonmuscle cells, including cell motility, cytokinesis, and phagocytosis. ${ }^{4,5}$ Recently, our research group showed that AIP1 and $\mathrm{ADF} /$ cofilin are both required for adherens junction remodeling in Drosophila eye epithelium. ${ }^{6}$

In muscle cells, actin-based thin filaments and myosin-based thick filaments are assembled into the repeated units of contractile apparatus known as sarcomeres. ${ }^{7}$ The actin filaments in sarcomeres are capped by CapZ at the barbed ends and by tropomodulin at the pointed ends; both of the capping proteins regulate the lengths of sarcomeric actin filaments. ${ }^{8,9}$ Because of this arrangement, actin filaments in muscle cells have been considered to be relatively stable and not to easily turn over. However, recent fluorescence recovery after photobleaching (FRAP) studies using microinjected fluorescently labeled actin or expressed GFP-actin suggest that the actin thin filaments undergo entire turnover at variable rates, from moderate to fast. ${ }^{10-15}$ Furthermore, a recent study using cultured neonatal rat cardiomyocytes showed that the turnover of sarcomeric thin filaments depends on the contractility of cardiomyocytes; functional knockdown of cofilin-1 and cofilin-2, two ADF/ cofilin isoforms present in mammalian muscle cells, led to disorganization of sarcomeric actin arrays. ${ }^{16}$ That study also provided evidence suggesting that $\mathrm{ADF} / \mathrm{cofilin}$ and contractility function together to disassemble or remove nonproductive actin filaments in developing cardiomyocytes. In accord with those findings, a $C f l 2$ (encoding cofilin-2) knockout mouse exhibited progressive sarcomeric disruption together with F-actin accumulation in the skeletal muscle. ${ }^{17}$

Patients identified with a CFL2 mutation exhibited nemaline myopathy, which is characterized by the presence of the rod-like nemaline bodies and areas of F-actin accumulation in the affected muscles. ${ }^{18,19}$ Moreover, the three major forms of congenital myopathy (ie, nemaline, actin, and intranuclear rod myopathies) all involve mutations in genes that encode actin-associated proteins, including the cofilin-2 (CFL2), $\alpha$-actin (ACTA1), $\alpha$ - and $\beta$-tropomyosin (TPM3 and TPM2), troponin $\mathrm{T}(T N N T 1)$, and nebulin $(N E B) \cdot{ }^{7,20}$ In particular, some of the mutations in ACTAl (skeletal muscle $\alpha$-actin) from patients were found to affect actin polymerization, but whether defects in actin dynamics are the direct cause of congenital myopathies remains to be elucidated. ${ }^{7,21}$

Genetic evidence for a role of AIP1 in muscle cells is available only from Caenorhabditis elegans. Loss of function of both UNC-78 and AIPL-1, two worm homologs of AIP1, resulted in severe disorganization of sarcomeres, with ectopic F-actin aggregates in body wall muscles. ${ }^{22-26}$ The mammalian homolog of AIP1 is encoded by the WD repeat domain 1 gene $(W d r l)$; both AIP1 and WDR1 are used as short names for this protein. A hypomorphic point mutation in $W d r l$ was shown to cause impairment of cytoskeletal response in neutrophils, resulting in autoinflammatory disease in mice, and a stronger insertional mutant allele resulted in embryonic lethality. ${ }^{27}$ However, complete deletion of
$W d r l$ has not been performed in vertebrates, and functional studies of AIP1 in vertebrate muscles are still lacking. Here, we report the first detailed genetic analysis of AIP1 in the vertebrate muscle. We generated conditional knockout (cKO) mice to specifically delete $W d r l$ in cardiomyocytes. These mice, which died at an early age, exhibited heart hypertrophy, electrocardiographic abnormalities, severe sarcomeric disruptions, and strong F-actin accumulations within muscle fibrils. Finally, in vitro actin depolymerization assay exhibited that mammalian AIP1 significantly enhances actin disassembly in the presence of cofilin, whereas AIP1 alone has no effect on actin depolymerization. Our findings indicate that AIP1-mediated actin disassembly plays essential roles during myocardial growth and maintenance in mice.

\section{Materials and Methods}

\section{Animal Studies}

The experimental animal facility of the Model Animal Research Center of Nanjing University has been accredited by the Association for Assessment and Accreditation of Laboratory Animal Care International (AAALAC), and the Institutional Animal Care and Use Committee (IACUC) of the Model Animal Research Institute of Nanjing University approved all animal protocols used in this study.

\section{Construction of Wdr1 Floxed Mice}

A region of approximately $10.2 \mathrm{~kb}$ used to construct the targeting vector was first subcloned from a bacterial artificial chromosome (BAC) clone using a homologous recombination-based technique. The BAC was subcloned into a PL253 backbone vector (approximately $5.4 \mathrm{~kb}$ ) containing an ampicillin selection cassette. A PL451 FRT-Neo-FRT/loxP cassette was inserted into the gene, and the $5^{\prime}$ homologous arm extended $4.1 \mathrm{~kb}$ to the $5^{\prime}$ end of the FRT-Neo-FRT/loxP cassette. The $3^{\prime}$ homologous arm extended $4.6 \mathrm{~kb}$ to the $3^{\prime}$ end of the single lox $P$ site. This single loxP site was inserted downstream of exon 4 , and the $F R T$ Neo-FRT/loxP cassette was inserted upstream of exon 4, so that exon 4 was the targeted region. The targeting vector was confirmed by restriction analysis after each modification step and by sequencing.

The targeting vector $(10 \mu \mathrm{g})$ was linearized by NotI endonuclease and then was electroporated into $129 \mathrm{~S} 6 / \mathrm{SvEvTac}-$ derived W4 embryonic stem cells. Positive embryonic stem cell clones were identified after PCR analysis and were further confirmed by Southern blotting analysis. In brief, DNA was digested with SpeI endonuclease and was electrophoretically separated on a $0.8 \%$ agarose gel. After transfer to a nylon membrane, the digested DNA was hybridized with a probe targeted against the $5^{\prime}$ homologous arm of the targeted locus. The expected DNA fragment sizes were $8588 \mathrm{bp}$ and $5781 \mathrm{bp}$ for the Wdrl Neo-flox conditional allele (Figure 1E). The probe primers were $5^{\prime}$-AGAACACTCCCTGGCTTCTAC-3' and $5^{\prime}$-CACCAGGAAAGCCTTATCAA- $3^{\prime}$ (forward and 
A

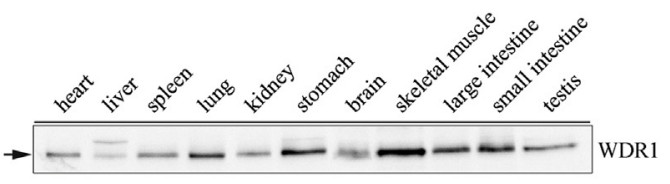

C

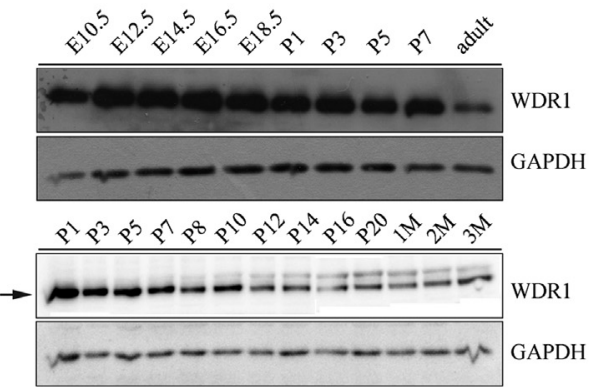

B

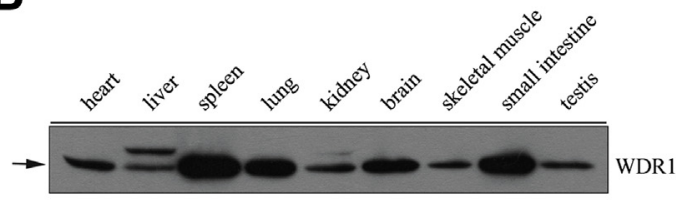

D

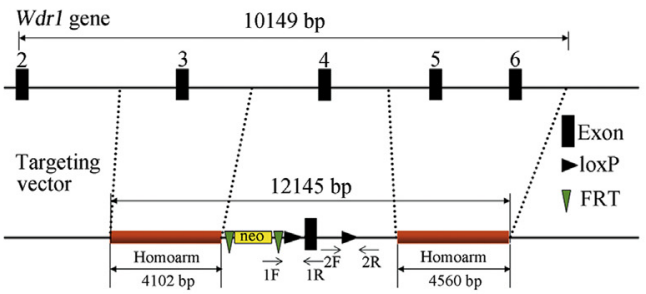

G
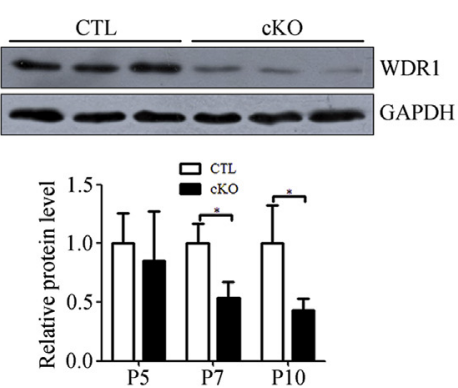

Figure 1 Generation of cardiomyocyte-specific Wdr1 deletion mice. A and B: Western blotting analysis of tissues dissected from 7-day-old (A) and 3-month-old (B) C57BL/6J mice. WDR1 was expressed in the heart of both pups and adults. C: Western blotting analysis of WDR1 expression levels in mouse heart from embryonic stages to adulthood. WDR1 protein levels were high during embryonic stages, but gradually decreased from birth to a constant level at adulthood. Target protein bands are indicated by an arrow (A-C). D: Schematic of targeting strategy to generate floxed Wdr1 allele (conditional targeted Wdr1 allele) as described under Materials and Methods. E: Southern blotting analysis. DNA isolated from electroporated embryonic stem cells was digested with SpeI and was hybridized with a probe targeted against the $5^{\prime}$ homologous arm. Expected sizes for the Wdr 1 Neo-flox conditional allele are shown. F: Genotyping by PCR analysis of DNA isolated from tail biopsy, showing wild-type, heterozygous, and homozygous genotypes for the conditionally targeted alleles. The first loxP $\left(5^{\prime}\right)$ site was detected using primers $1 \mathrm{~F}$ and $1 \mathrm{R}$ and the second $\left(3^{\prime}\right)$ site using primers $2 \mathrm{~F}$ and $2 \mathrm{R}$ (as shown in $\mathbf{D}$ and listed under Materials and Methods). The $5^{\prime}$ floxed allele was $334 \mathrm{bp}$ and the wild-type was $230 \mathrm{bp}$; the $3^{\prime}$ floxed allele was $317 \mathrm{bp}$ and the wild-type was $219 \mathrm{bp}$. G: Detection of WDR1 by Western blotting analysis in protein extracted from hearts of Wdr1 $1^{f / f}(\mathrm{CTL})$ and Wdr1 ${ }^{f / f} ; \alpha \mathrm{MHC}-\mathrm{Cre}(\mathrm{cKO})$ mice at P10 revealed significantly reduced levels of WDR1 in cK0 mice. Quantification of WDR1 deletion efficiency at P5, P7, and P10 revealed a slight but nonsignificant reduction in cK0 mice at P5 and a significant reduction at P7, with further reduction at P10. Data are expressed as means \pm SEM. $n=3$ mice per group. ${ }^{*} P<0.05$.

reverse, respectively). The correctly targeted embryonic stem cells were microinjected into BALB/c mouse blastocysts. The resulting chimeras with a high-percentage of gray coat color were mated to $\mathrm{C} 57 \mathrm{BL} / 6 \mathrm{~J}$ mice to generate heterozygous Wdrl Neo-flox/+ mice. Tail DNA was analyzed for heterozygosity of the $W d r l$ Neo-flox conditional allele $\left(W d r I^{\text {neo-f/+ }}\right.$ ) using the second $\operatorname{lox} P\left(3^{\prime}\right)$, with forward primer (2F) 5'-GGACCTTCTAAGCAGTTACAACC-3' and reverse primer (2R) 5' $5^{\prime}$ TTGCACAGAGGTGAATGACAGAG- $3^{\prime}$, to include the $3^{\prime} \operatorname{lox} P$ site present in the $W d r l$ Neo-flox conditional allele. With the addition of the loxP site, the amplified floxed allele was $317 \mathrm{bp}$, whereas the wildtype allele was $219 \mathrm{bp}$.

To remove the FRT-Neo-FRT/loxP cassette, we bred the $W d r r^{\text {neo-ff/ }}$ mice with Flp recombinase mice that ubiquitously expressed the Flp recombinase in all cells of the embryo. ${ }^{28}$ Removal of the FRT-Neo-FRT/loxP cassette from the $W d r l$ Neo-flox/+ allele to obtain the $W d r l$ floxed allele was confirmed by PCR analysis (data not shown).
The heterozygotic $W d r l$ flox/+ mice $\left(W d r 1^{f /+}\right)$ were backcrossed to C57BL/6J mice and then bred to obtain the homozygous floxed mice $\left(W d r f^{f f}\right)$. In addition to use of the second lox $P\left(3^{\prime}\right)$ for genotyping, the genotype was alternatively analyzed for conditional $W d r l$ floxed allele using the first loxP $\left(5^{\prime}\right)$, with forward primer (1F) $5^{\prime}$-GCTATAAATGAGGTCCTGATGAG- $3^{\prime}$ and reverse primer (1R) $5^{\prime}-$ TCAGGCTTACAAAGCTCACATG- $3^{\prime}$, to include $5^{\prime}$ loxP site present in the $W d r l$ floxed allele. The amplified floxed allele was $334 \mathrm{bp}$, whereas the wild-type allele was $230 \mathrm{bp}$ (Figure 1F). Routine genotyping was achieved on genomic DNA isolated from tail biopsy of mice.

\section{Generation of Cardiomyocyte-Specific Wdr1 Deletion Mice}

$W d r 1^{f / f} ; \alpha M H C-C r e$ Mice

$W d r l^{f f f}$ mice were bred with $\mathrm{Tg}$ (Myh6-cre) transgenic mice (aMHC-Cre) obtained from Dr. Xiao Yang at the Beijing 
Institute of Biotechnology, China, to generate $\mathrm{Wdr} \mathrm{r}^{\mathrm{fl}+}$; aMHC-Cre mice, which were then bred with $W d r l^{f f f}$ mice to generate $W d r I^{f f f} ; \alpha \mathrm{MHC}-\mathrm{Cre}$ mice. The primers used to detect $\alpha$ MHC-Cre were forward, 5'-AATGCTTCTGTCCGTTTGC-3', and reverse, 5'-ACCAGAGTCATCCTTAGCG- $3^{\prime}$. A 712-bp band is present in the $\alpha \mathrm{MHC}-\mathrm{Cre}$ transgenic mice.

\section{$W d r 1^{f / f} ; \alpha M H C-M e r C r e M e r$ iKO Mice}

$W d r 1^{f / f}$ mice were bred with $\mathrm{Tg}\left(\mathrm{Myh} 6-\mathrm{cre} / \mathrm{Esr} 1^{*}\right) 1 \mathrm{Jmk}$ transgenic mice (hereafter, $\alpha$ MHC-MerCreMer) obtained from the Jackson Laboratory (Bar Harbor, ME) to generate $W d r l^{f /+} ; \alpha \mathrm{MHC}-\mathrm{MerCreMer}$ mice inducible knockout (iKO) mice. $W d r 1^{f /+} ; \alpha$ MHC-MerCreMer mice were then bred with $W d r r^{f f f}$ mice to generate $W d r l^{f f f} ; \alpha M H C-M e r C r e M e r$ mice. The primers used to detect $\alpha \mathrm{MHC}-\mathrm{MerCreMer}$ mice were the same as used for the $\alpha \mathrm{MHC}-\mathrm{Cre}$ allele. At 2 months of age, $W d r l^{f f f} ; \propto \mathrm{MHC}-\mathrm{MerCreMer}$ mice and their $W d r{ }^{f f f}$ littermates were treated with tamoxifen (T5648-5G; Sigma-Aldrich, St. Louis, MO) dissolved in oil by intraperitoneal injection once a day for five consecutive days at a dosage of $20 \mathrm{mg} / \mathrm{kg}$ per day.

\section{Histological Analysis}

$\mathrm{H} \& \mathrm{E}$ staining was performed using standard protocols. In brief, heart samples were first washed with cold PBS and then fixed in $4 \%$ paraformaldehyde at $4^{\circ} \mathrm{C}$. The samples were processed successively by i) one 20-minute wash in PBS; ii) six 1-hour incubations in a graded ethanol series $(70 \%, 85 \%$, $95 \%, 95 \%, 100 \%$, and $100 \%$ ethanol) at room temperature; iii) three 10-minute incubations in xylene at room temperature; iv) one 30-minute incubation in paraffin-xylene (1:1) at $65^{\circ} \mathrm{C}$; and v) three 1-hour incubations in fresh paraffin at $65^{\circ} \mathrm{C}$. The processed samples were then embedded in paraffin, sectioned in 6- $\mu \mathrm{m}$ thickness, and stained with $\mathrm{H} \& \mathrm{E}$.

\section{RT-qPCR}

Total RNA was extracted from ventricular myocardium using TRIzol reagent (Life Technologies, Carlsbad, CA) according to the manufacturer's protocol. One microgram of total RNA from each specimen was reverse-transcribed to cDNA using a Promega (Madison, WI) Reverse Transcription System kit. The cDNA was then diluted fivefold with Milli-Q ultrapure water (EMD Millipore, Billerica, MA). Quantitative real-time reverse transcription PCR (RT-qPCR) was performed with an ABI StepOnePlus instrument (Life Technologies) using Platinum SYBR Green qPCR SuperMix-UDG with ROX (Life Technologies), $250 \mathrm{nmol}$ for forward and reverse primers, and $1 \mu \mathrm{L}$ diluted cDNA in a total volume of $10 \mu \mathrm{L}$ reaction system. All of the primers were designed with Primer Premier 5.0 (PREMIER Biosoft, Palo Alto, CA) or according to published sources. Primers for ANP, BNP, and the internal control GAPDH were as published. ${ }^{29}$ The other primers were as follows: $\alpha$-MHC forward 5'-AGTGCTTCGTGCCTGATGA-3' and reverse $5^{\prime}$-AACTTGGGTGGGTTCTGCT-3'; $\beta$-MHC primer forward $5^{\prime}$-GTCCAAGTTCCGCAAGGT- $3^{\prime}$ and reverse $5^{\prime}$-GGAGCTGGGTAGCACAAGA-3'; Actal forward $5^{\prime}$-AGAGTCAGAGCAGCAGAAACTAG- $3^{\prime}$ and reverse $5^{\prime}$-CCACGATGGATGGGAACAC-3'; Acta2 forward $5^{\prime}$-AGGAACCCTGAGACGCTGCT- $3^{\prime}$ and reverse 5'-CCATTCCAACCATTACTCCC-3'; Actcl forward 5'-CTCCAACTGACCCCGTCCAT- $3^{\prime}$ and reverse $5^{\prime}$-AAAGCGGTGGTCTCCTCGTC- ${ }^{\prime}$; Actb forward 5'-CACCGCAAGTGCTTCTAGG- $3^{\prime}$ and reverse $5^{\prime}$-GCCATGCCAATGTTGTCTC-3'; Actgl forward 5'-CAGTCTAACAGGGTGGGAAAG- $3^{\prime}$ and reverse $5^{\prime}$-CCAACTCAAGGCAA-CTAACAA-3' The PCR program used was $95^{\circ} \mathrm{C}$ for 15 seconds, followed by 40 cycles of $95^{\circ} \mathrm{C}$ for 5 seconds and $60^{\circ} \mathrm{C}$ for 31 seconds. The relative amount of target gene expression in $\mathrm{cKO}$ mice was calculated as fold change relative to littermate controls, normalized to GAPDH.

\section{Electron Microscopy Study}

Mouse heart was perfused with $2.5 \%$ glutaraldehyde in 0.1 $\mathrm{mol} / \mathrm{L}$ sodium phosphate ( $\mathrm{pH} 7.4$ ), for 10 to 15 minutes. The heart was isolated, cut into small pieces $\left(<2 \mathrm{~mm}^{3}\right)$, and postfixed overnight at $4^{\circ} \mathrm{C}$ in $2.5 \%$ glutaraldehyde in 0.1 $\mathrm{mol} / \mathrm{L}$ sodium phosphate. After postfixation, the specimens were processed at the College of Life Science, Nanjing Agricultural University (Nanjing, China).

\section{Immunofluorescence}

For immunofluorescence studies, 8 - $\mu$ m-thick frozen sections were double-stained with mouse monoclonal anti- $\alpha$ actinin (sarcomeric) (1:100 dilution; A7811; Sigma-Aldrich) and tetramethylrhodamine B isothiocyanate (TRITC)conjugated phalloidin 546 (p1951; Sigma-Aldrich), mouse monoclonal anti-purified tropomodulin-1 (Tmod1) (1:100 dilution; TA503170; OriGene Technologies, Rockville, MD) and TRITC-conjugated phalloidin 546, or rabbit polyclonal anti-total cofilin (1:50 dilution; BS2183; Bioworld Technology, St. Louis Park, MN) and TRITCconjugated phalloidin 546. Secondary antibodies were Alexa Fluor 488-conjugated mouse IgG (115-545-166; Jackson ImmunoResearch Laboratories, West Grove, PA) and Alexa Fluor 488-conjugated anti-rabbit IgG (111-545144; Jackson ImmunoResearch Laboratories). For phalloidin staining, frozen heart sections were incubated with TRITC-conjugated phalloidin and DAPI $(1 \mu \mathrm{g} / \mathrm{mL}$ working concentration) (D6584; Sangon Biotech, Shanghai, China) for 2 hours at room temperature. Fluorescence microscopy images were obtained with a research fluorescence microscope (Olympus, Tokyo, Japan; Leica Microsystems, Wetzlar, Germany) equipped with a digital camera.

\section{Western Blotting}

Heart tissues were frozen in liquid nitrogen immediately after dissection and stored at $-80^{\circ} \mathrm{C}$ until analysis. Tissue 
lysates were prepared in lysis buffer $[20 \mathrm{mmol} / \mathrm{L}$ Tris, 150 $\mathrm{mmol} / \mathrm{L} \mathrm{NaCl}, 10 \%$ glycerol, $20 \mathrm{mmol} / \mathrm{L}$ glycerophosphate, $1 \% \mathrm{NP} 40,5 \mathrm{mmol} / \mathrm{L}$ EDTA, $0.5 \mathrm{mmol} / \mathrm{L}$ EGTA, $1 \mathrm{mmol} / \mathrm{L}$ $\mathrm{Na}_{3} \mathrm{VO}_{4}, \quad 0.5 \mathrm{mmol} / \mathrm{L}$ phenylmethanesulfonyl fluoride (PMSF), $1 \mathrm{mmol} / \mathrm{L}$ benzamidine, $1 \mathrm{mmol} / \mathrm{L}$ dithiothreitol, $50 \mathrm{mmol} / \mathrm{L} \mathrm{NaF}$, and $4 \mathrm{~mol} / \mathrm{L}$ leupeptin at $\mathrm{pH} 8.0]$. Samples were resolved by SDS-PAGE and were transferred to polyvinylidene difluoride membranes (EMD Millipore). Membranes were blocked with 5\% nonfat milk in TBST $(50 \mathrm{mmol} / \mathrm{L}$ Tris, $150 \mathrm{mmol} / \mathrm{L} \mathrm{NaCl}$, and 0.5 $\mathrm{mmol} / \mathrm{L}$ Tween 20 at $\mathrm{pH} 7.5$ ) and then incubated with primary antibodies overnight at $4^{\circ} \mathrm{C}$. Transferred proteins were probed with antibodies against WDR1 (1:1000 dilution; 3676-1-AP; Proteintech Group, Chicago, IL), pan-actin (1:3000 dilution; MS-1295-P1ABX; Thermo Fisher Scientific, Waltham, MA), Tmod1 (1:1000 dilution; TA503170; OriGene Technologies), $\alpha$-sarcomeric actinin (1:300 dilution; A7811; Abcam, Cambridge, MA), total cofilin (1:500 dilution; 21164-1; Signalway Antibody, College Park, MD), tropomyosin (1:1000 dilution; $\mathrm{CH} 1$; Abcam), cardiac troponin T (cTnT) (1:500 dilution; CT3; Developmental Studies Hybridoma Bank, Iowa City, IA), cardiac troponin I (cTnI) (1:500 dilution; Chemicon MAB1691; EMD Millipore), and GAPDH (1:20,000 dilution; AP0063; Bioworld Technology) and then were visualized using enhanced chemiluminescence. Quantification of protein levels normalized to GAPDH was performed using ImageJ software version 1.44 (NIH, Bethesda, MD). G-actin/F-actin assay was performed using a kit (BK037; Cytoskeleton, Denver, CO).

\section{Expression and Purification of Recombinant GST-WDR1 and GST-Cofilin-2 Fusion Proteins}

The entire coding region of $W d r l$ (1821 bp) or $C f l 2$ (cofilin2; $501 \mathrm{bp)} \mathrm{cDNA} \mathrm{was} \mathrm{cloned} \mathrm{from} \mathrm{E12.5} \mathrm{heart} \mathrm{cDNA,} \mathrm{and}$ then was cloned into PGEX-4T-1 vector (a gift from Dr. Ying $\mathrm{Xu}$, Nanjing University, China) between EcoRI and XhoI. The resultant vector expresses fusion proteins of WDR1 or cofilin-2 with GST and thrombin at the N-terminus. Escherichia coli strain BL21 (DE3) (a gift from Dr. Shuai Chen, Nanjing University, China) was transformed with the expression vector and cultured in LB medium containing $50 \mathrm{mg} / \mathrm{mL}$ ampicillin at $37^{\circ} \mathrm{C}$ until optical density at $600 \mathrm{~nm}$ reached 0.6 to 0.8 . The culture was then cooled, and WDR1 protein expression was induced by adding $0.4 \mathrm{mmol} / \mathrm{L}$ isopropyl $\beta$-D-thiogalactopyranoside 8 hours at $15^{\circ} \mathrm{C}$; expession of cofilin-2 protein was induced by adding $0.05 \mathrm{mmol} / \mathrm{L} \beta$-D-thiogalactopyranoside 5 hours at $22^{\circ} \mathrm{C}$. The cells were harvested by centrifugation at $5000 \times g$ for 15 minutes, disrupted by sonication in lysis buffer $(0.2 \mathrm{mmol} / \mathrm{L}$ PMSF, $1 \mu \mathrm{g} / \mathrm{mL}$ leupeptin, $5 \mathrm{mmol} / \mathrm{L}$ dithiothreitol, $0.5 \mathrm{mmol} / \mathrm{L}$ EDTA, $0.5 \mathrm{mmol} / \mathrm{L}$ EGTA, and $10 \mathrm{U} / \mathrm{mL}$ DNase I), and lysed for 30 minutes on ice. The homogenates were centrifuged at $20,000 \times g$ for $15 \mathrm{mi}-$ nutes, and the supernatants were applied to a GST fusion protein purification kit (L00207; GenScript, Piscataway, NJ). Glutathione resin that bonded GST-WDR1 or GST-cofilin-2 on columns was washed three times with washing buffer $(0.2 \mathrm{mmol} / \mathrm{L}$ PMSF, $1 \mu \mathrm{g} / \mathrm{mL}$ leupeptin, 5 $\mathrm{mmol} / \mathrm{L}$ dithiothreitol, $0.5 \mathrm{mmol} / \mathrm{L}$ EDTA, $0.5 \mathrm{mmol} / \mathrm{L}$ EGTA, $10 \mathrm{U} / \mathrm{mL}$ DNase I). Bound GST-cofilin-2 proteins were eluted with elution buffer $(1 \mathrm{mmol} / \mathrm{L}$ benzamidine, 1 $\mu \mathrm{g} / \mathrm{mL}$ leupeptin, $0.2 \mathrm{mmol} / \mathrm{L}$ PMSF, $0.1 \mathrm{mmol} / \mathrm{L}$ EGTA, 20 $\mathrm{mmol} / \mathrm{L}$ reduced glutathione, $10 \%$ glycerol, and $100 \mathrm{mmol} / \mathrm{L}$ Tris- $\mathrm{HCl}$ at $\mathrm{pH}$ 8.0). Bound GST-WDR1 proteins were eluted with elution buffer $(1 \mathrm{mmol} / \mathrm{L}$ benzamidine, $1 \mu \mathrm{g} / \mathrm{mL}$ leupeptin, $0.2 \mathrm{mmol} / \mathrm{L}$ PMSF, $0.1 \mathrm{mmol} / \mathrm{L}$ EGTA, $40 \mathrm{mmol} / \mathrm{L}$ reduced glutathione, $10 \%$ glycerol, $100 \mathrm{mmol} / \mathrm{L}$ Tris- $\mathrm{HCl}$ (pH 8.0), $0.1 \mathrm{~mol} / \mathrm{L} \mathrm{NaCl}$, and $0.1 \%$ Triton X-100 surfactant). Fractions containing pure GST-WDR1 or GSTcofilin-2 were dialyzed against $5 \mathrm{mmol} / \mathrm{L}$ Tris- $\mathrm{HCl}$ at $\mathrm{pH} 8.0$ overnight at $4^{\circ} \mathrm{C}$ and stored at $-80^{\circ} \mathrm{C}$.

\section{Assay for Actin Depolymerization}

In vitro actin depolymerization assay was performed using an Actin Polymerization Biochem kit (BK003; Cytoskeleton). In brief, pyrene-labeled F-actin stock was prepared by incubating $0.4 \mathrm{mg}$ pyrene G-actin, diluted in $400 \mu \mathrm{L}$ G buffer $(5 \mathrm{mmol} / \mathrm{L}$ Tris-HCl at $\mathrm{pH} 8.0,0.2 \mathrm{mmol} / \mathrm{L} \mathrm{CaCl}_{2}$, and $0.2 \mathrm{mmol} / \mathrm{L} \mathrm{ATP)}$ with $10 \mu \mathrm{L} 10 \times$ actin polymerization buffer $(0.25 \times$ final strength) $(500 \mathrm{mmol} / \mathrm{L} \mathrm{KCl}, 20 \mathrm{mmol} / \mathrm{L} \mathrm{MgCl} 2,0.05 \mathrm{~mol} / \mathrm{L}$ guanidine carbonate, and $10 \mathrm{mmol} / \mathrm{L} \mathrm{ATP}$ ) at room temperature for 1 hour. The F-actin stock was then diluted to $0.2 \mathrm{mg} /$ $\mathrm{mL}$ by adding $1.6 \mathrm{~mL}$ of $\mathrm{G}$ buffer; and $200 \mu \mathrm{L}$ diluted F-actin stock was pipetted into wells of 96-well plates. The depolymerization of pyrene F-actin was measured by fluorescence spectrometer counts at an excitation wavelength of $360 \mathrm{~nm}$ with emission at $410 \mathrm{~nm}$ every 30 seconds for 1 hour. The Factin depolymerization was performed by adding the same volume of test buffer $(5 \mathrm{mmol} / \mathrm{L}$ Tris- $\mathrm{HCl}$ at $\mathrm{pH} 8.0,3.78$ $\mu \mathrm{mol} / \mathrm{L}$ F-actin), GST-cofilin-2 (3.78 $\mu \mathrm{mol} / \mathrm{L}$ F-actin, 1.89 $\mu \mathrm{mol} / \mathrm{L}$ GST-cofilin-2), GST-WDR1 (3.78 $\mu \mathrm{mol} / \mathrm{L}$ F-actin, $0.38 \mu \mathrm{mol} / \mathrm{L}$ GST-WDR1), GST-cofilin-2 + GST-WDR1 (3.78 $\mu \mathrm{mol} / \mathrm{L}$ F-actin, $1.89 \mu \mathrm{mol} / \mathrm{L} \mathrm{GST}-$ cofilin- 2 , and 0.38 $\mu \mathrm{mol} / \mathrm{L}$ GST-WDR1) into wells containing $200 \mu \mathrm{L}$ diluted F-actin stock.

\section{Echocardiography Assessment of Cardiac Function}

Mice were anesthetized intraperitoneally with $0.4 \mathrm{mg} / \mathrm{g}$ of locally formulated avertin echocardiography was performed with a Vevo 770 UBM ultrasound biomicroscopy system (VisualSonics, Toronto, ON, Canada). This system has a single-element mechanical transducer with a center frequency of $30 \mathrm{MHz}$ and a frame rate of $30 \mathrm{~Hz}$ using M-mode and two-dimensional measurements to assess systolic function. The internal diameter of the left ventricle (LV) in the short-axis plane was measured at end diastole and end systole from M-mode recordings. The interventricular and LV posterior wall thicknesses were measured at end 
A

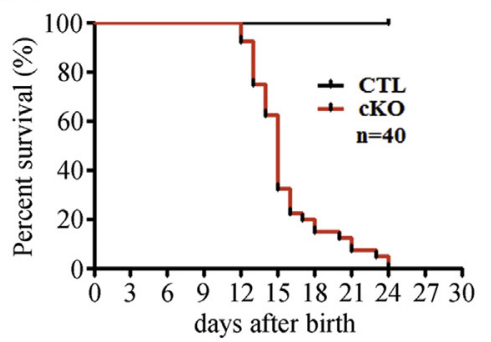

B

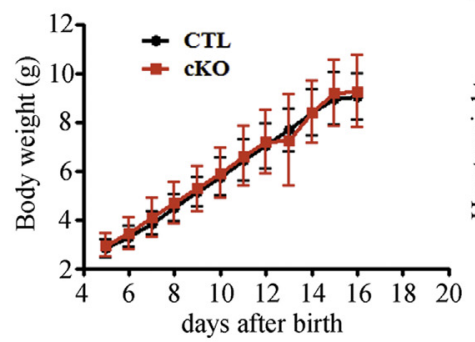

C

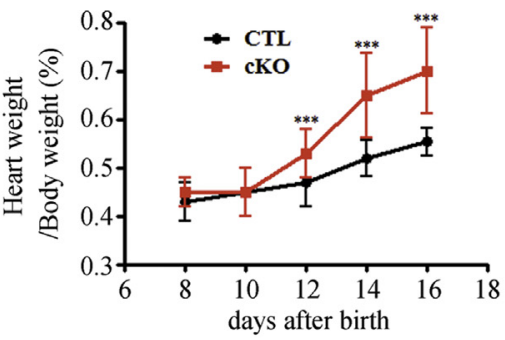

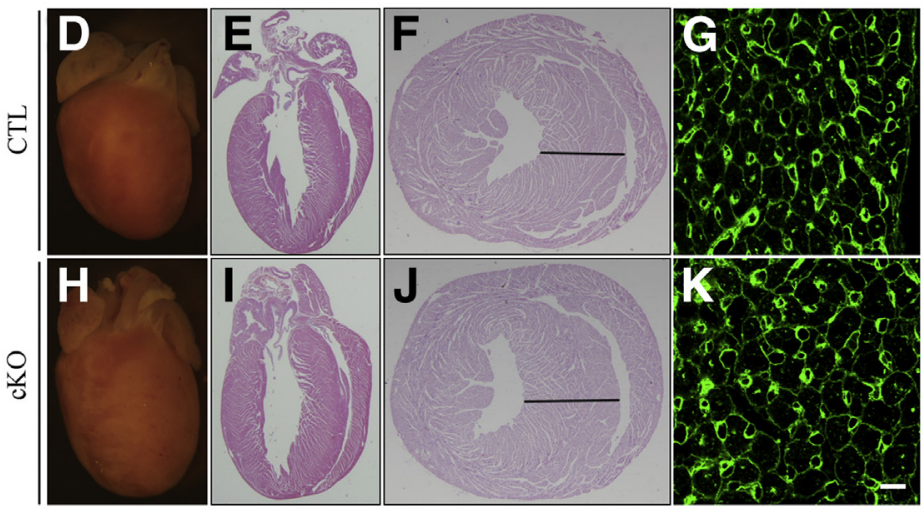
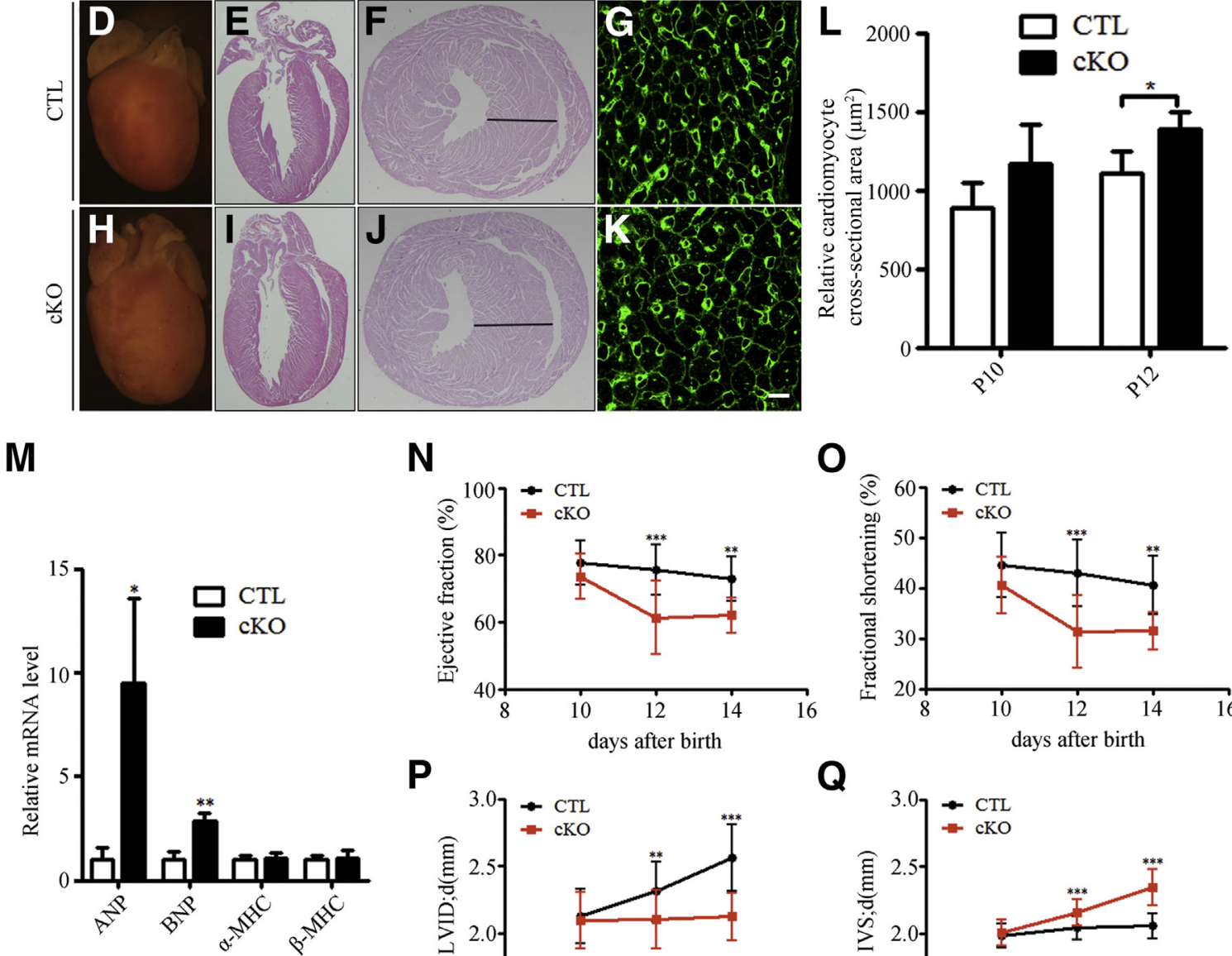

$\mathbf{N}$

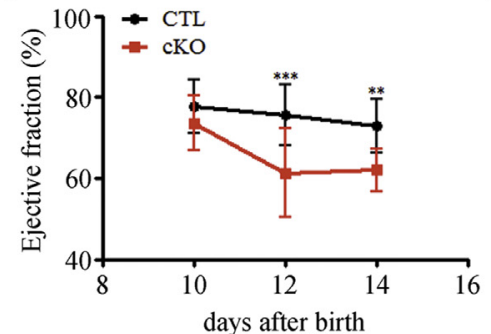

0

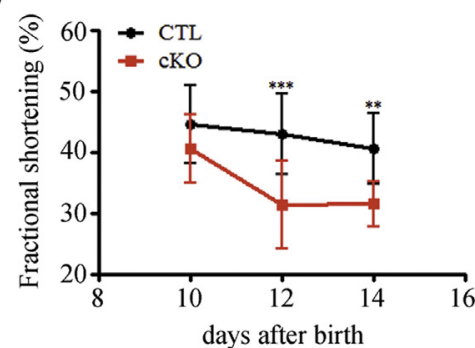

$\mathbf{P}$

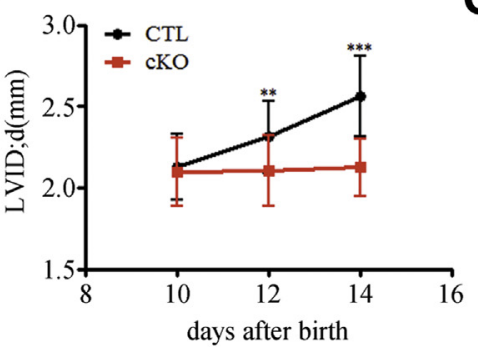

Q

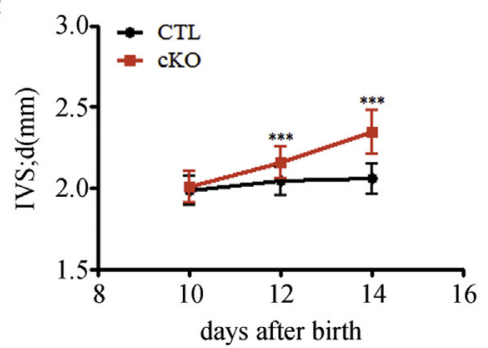

Figure 2 Wdr1 cK0 mice exhibit heart hypertrophy, impaired heart contraction function, and early lethality. A: The cK0 mice began to die at P13, and none survived past P24. B: Body weight did not differ between CTL and CKO mice from birth to death. C: The heart weight/body weight ratio of cK0 and CTL mice was similar at P10. Beginning at P12, however, the heart weight/body weight ratio of cK0 mice was significantly increased. D and $\mathbf{H : ~ T h e ~ h e a r t ~ i n ~ c K 0 ~ m i c e ~ ( H ) ~ w a s ~}$ larger than that of CTL mice (D) at P12. E, F, I, and J: Histological analysis of heart samples at P12 (longitudinal sections, E and I; cross sections, F and J) shows that the heart of CKO mice was larger than that of CTL mice. H\&E stain. $\mathbf{G}$ and $\mathbf{K}$ : Immunofluorescence staining (wheat germ agglutinin) on heart cross sections from CTL (G) and CKO (K) mice at P12 revealed increased cardiomyocyte size in cK0 mice, compared with CTL. L: Cardiomyocyte cross-sectional area calculated by ImageJ version 1.44, which started to increase in CK0 mice at P10, was significantly greater at P12 and P14, compared with CTL. M: mRNA levels of four heart remodeling markers at P10 were determined by qPCR. Expression levels of ANP and BNP were increased in cKO mice, compared with CTL, but $\alpha-$ MHC and $\beta-M H C$ levels were unchanged. $\mathbf{N}-\mathbf{Q}$ : Echocardiographic study showed that ejective fraction $(\mathbf{N})$, fractional shortening $(\mathbf{0})$, and left ventricular internal dimension in diastole $(\mathbf{P})$ began to decrease and interventricular septum in diastole $(\mathbf{Q})$ began to increase at P10 in cK0 mice, compared with CTL. Data are expressed as means \pm SEM. $n=3$ mice per group. ${ }^{*} P<0.05,{ }^{*} P<0.01$, and ${ }^{* *} * P<0.001$. Scale bar $=10 \mu \mathrm{m}$.

diastole. LV fractional shortening (FS) and LV ejection fraction (EF), as an index of LV systolic function, were calculated according to the manufacturer's instructions for the Vevo 770 UBM system.

\section{Statistical Analysis}

Statistical calculations were performed using unpaired twotailed Student's $t$-tests. Data are expressed as means \pm 
SEM. The threshold for statistical significance was set at $P<0.05$.

\section{Results}

\section{Generation of Cardiomyocyte-Specific Wdr1 Deletion Mice}

We first determined the expression pattern of WDR1 in young and adult mice by Western blotting and found that WDR1 was expressed ubiquitously, at varied levels in different tissues (Figure 1, A and B). Next, we performed a temporal analysis of WDR1 expression levels in the heart from embryonic stage to adulthood [ie, from embryonic day 10.5 (E10.5) to postnatal day 20 (P20) to 3 months]; the protein level was highest during the embryonic stage, but became gradually decreased to a constant level from birth to adulthood (Figure 1C).

We generated $W d r l$ cKO mice by a recombination-based method. A FRT-Neo-FRT/loxP cassette was inserted upstream of exon 4 and a loxP site was inserted downstream of exon 4, to generate a conditional mutant allele. This allows for excision of $W d r l$ in a tissue-specific manner (Figure 1D). The targeting vector was electroporated into 129S6/SvEvTac-derived W4 embryonic stem cells under G418 selection, and clones that underwent proper homologous recombination were identified and confirmed by genomic PCR (data not shown) and Southern blotting (Figure 1E). The correctly targeted embryonic stem cells were microinjected into BALB/c blastocysts, and chimeras that transmitted the $W d r l$ Neo-flox/+ allele through the germline were identified. Resulting chimeras were crossed with Flp recombinase mice to remove the FRT-Neo-FRT/loxP cassettes and then were mated to C57BL/6J mice to generate heterozygous $\mathrm{Wdr} \mathrm{I}^{\mathrm{f} /+}$ mice. To disrupt gene expression of $W d r l$, exon 4 of $W d r l$ was flanked by loxP sequences (floxed) for Cre recombinasemediated exon 4 deletion (Figure 1D).
To specifically ablate $W d r l$ in cardiomyocytes, homozygous $W d r l^{f f f}$ mice were bred with $\alpha$ MHC-Cre transgenic mice, which enabled Cre recombinase to be expressed specifically in cardiomyocytes, starting at $\mathrm{P} 3$ and reaching the maximal expressed level at P7 (unpublished data and Refs. $\left.{ }^{30,31}\right)$. The heterozygous $W d r r^{f /+} ; \alpha$ MHC-Cre mice were healthy and viable, and were bred with $W d r f^{f f f}$ mice to obtain homozygous $W d r l$ deletion mice $\left(W d r 1^{f f f} ; \alpha \mathrm{MHC}\right.$ $\mathrm{Cre}$, hereafter referred to as $W d r l \mathrm{cKO}$ ) (Figure $1 \mathrm{~F}$ ). The $W d r l$ excision in the $W d r l$ cKO mice was confirmed by Western blotting analysis. At P5, the protein levels of WDR1 in the heart of cKO mice were similar to those of control mice. However, WDR1 levels were profoundly reduced in cKO hearts at P7 and P10, compared with control (Figure 1G).

\section{Wdr1 cKO Mice Exhibit Heart Hypertrophy, Impaired Heart Contraction Function, and Early Lethality}

Wdrl cKO mice were born at Mendelian ratios and were indistinguishable from their control (CTL) littermates at birth, and all survived until P13. From P13, Wdrl cKO mice began to die, often of sudden death, and none survived past P24 (Figure 2A). Body weight was similar in CTL and cKO mice from birth to death (Figure 2B). At P10, the heart weight/body weight ratio, expressed as a percentage, was unchanged in $W d r l$ cKO heart $(0.45 \% \pm 0.05)$, relative to CTL $(0.45 \% \pm 0.05)$; beginning at P12, however, a significant increase in heart weight/body weight ratio was observed in $W d r l$ cKO mice $(0.53 \% \pm 0.05)$, relative to CTL $(0.47 \% \pm 0.05)(P<0.001)$, (Figure 2C).

Histological analysis revealed that, at P10, the heart of $W d r l$ cKO mice was similar to that of CTL mice (data not shown), but was larger than CTL at P12 (Figure 2, D-F and $\mathrm{H}-\mathrm{J})$. Microscopic analysis of cardiomyocytes stained with fluorochrome-labeled wheat germ agglutinin revealed no significant increase in cross-sectional surface area at P10.

Table 1 Echocardiographic Study of Wdr1 cK0 Mouse Pups

\begin{tabular}{|c|c|c|c|c|c|c|}
\hline \multirow[b]{2}{*}{ Measurement } & \multicolumn{2}{|l|}{ P10 } & \multicolumn{2}{|l|}{$\mathrm{P} 12$} & \multicolumn{2}{|l|}{ P14 } \\
\hline & $\mathrm{CTL}(n=16)$ & $\operatorname{cKO}(n=21)$ & $\operatorname{CTL}(n=22)$ & $\operatorname{cKO}(n=28)$ & $\operatorname{CTL}(n=8)$ & $\operatorname{cKO}(n=10)$ \\
\hline IVS, d (mm) & $0.49 \pm 0.089$ & $0.51 \pm 0.095$ & $0.54 \pm 0.087$ & $0.66 \pm 0.095^{* * *}$ & $0.56 \pm 0.096$ & $0.85 \pm 0.136^{* * *}$ \\
\hline LVPW, d (mm) & $0.52 \pm 0.116$ & $0.51 \pm 0.064$ & $0.55 \pm 0.07$ & $0.62 \pm 0.097 * *$ & $0.6 \pm 0.038$ & $0.69 \pm 0.039 * * *$ \\
\hline IVS, s (mm) & $0.68 \pm 0.162$ & $0.67 \pm 0.133$ & $0.8 \pm 0.111$ & $0.82 \pm 0.112$ & $0.82 \pm 0.078$ & $1.03 \pm 0.12^{* * *}$ \\
\hline LVID, s (mm) & $1.19 \pm 0.217$ & $1.25 \pm 0.221$ & $1.32 \pm 0.196$ & $1.45 \pm 0.26$ & $1.53 \pm 0.248$ & $1.45 \pm 0.111$ \\
\hline LV Vol, s (mm) & $3.52 \pm 1.486$ & $4.02 \pm 1.847$ & $4.57 \pm 1.723$ & $5.9 \pm 3.051$ & $6.71 \pm 2.643$ & $5.64 \pm 1.119$ \\
\hline $\mathrm{EF}(\%)$ & $77.71 \pm 6.557$ & $73.54 \pm 6.774$ & $75.62 \pm 7.405$ & $61.26 \pm 10.909 * * *$ & $72.88 \pm 6.668$ & $61.99 \pm 5.345^{* *}$ \\
\hline FS (\%) & $44.54 \pm 6.355$ & $40.57 \pm 5.636$ & $42.87 \pm 6.575$ & $31.39 \pm 7.201^{* * *}$ & $40.49 \pm 5.818$ & $31.46 \pm 3.718^{* *}$ \\
\hline LV mass (mg) & $22.57 \pm 4.981$ & $22.21 \pm 4.42$ & $28.83 \pm 6.653$ & $31.16 \pm 5.331$ & $36.54 \pm 5.889$ & $41.79 \pm 7.663$ \\
\hline
\end{tabular}

Data are expressed as means \pm SEM.

${ }^{*} P<0.05,{ }^{*} P<0.01$, and ${ }^{* * *} P<0.001$ versus CTL.

d, diastolic; EF, ejection fraction; FS, fractional shortening; IVS, interventricular septum; LV, left ventricle; LVID, left ventricular internal dimension; LVPW, left ventricular posterior wall; s, systolic. 


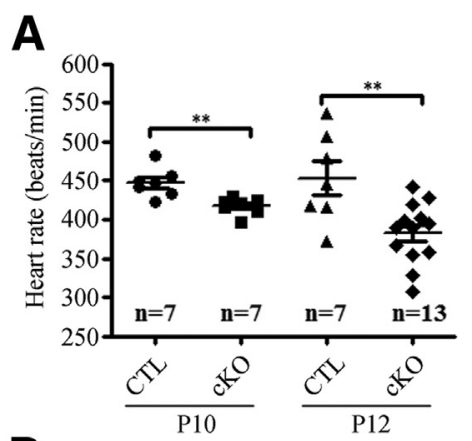

D

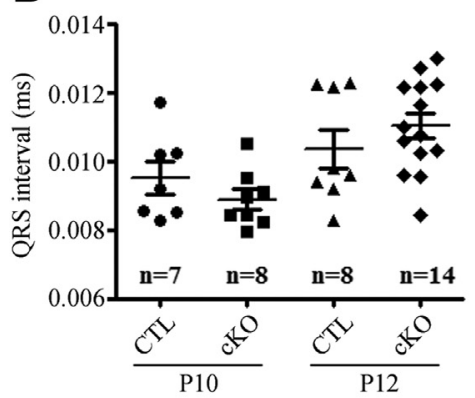

G

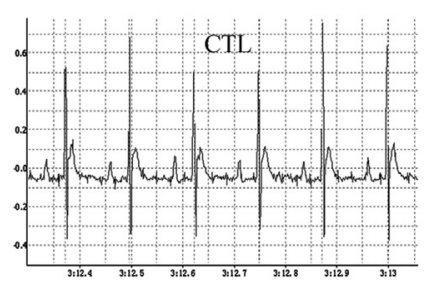

B

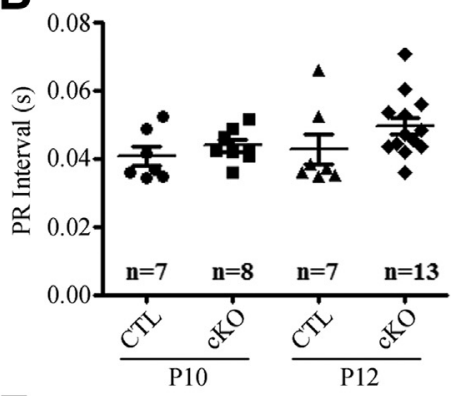

E

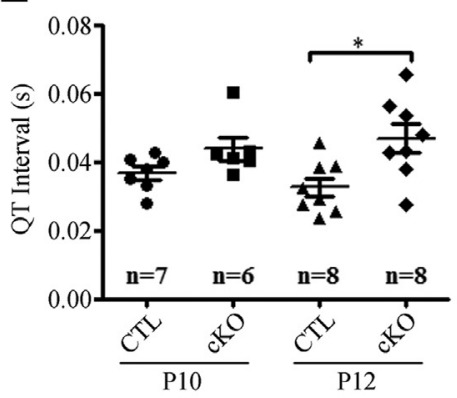

H

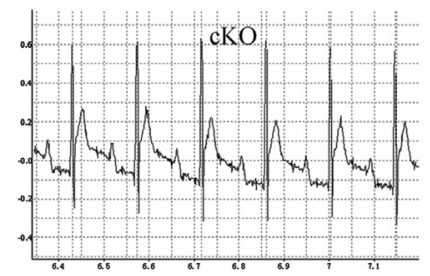

C

F
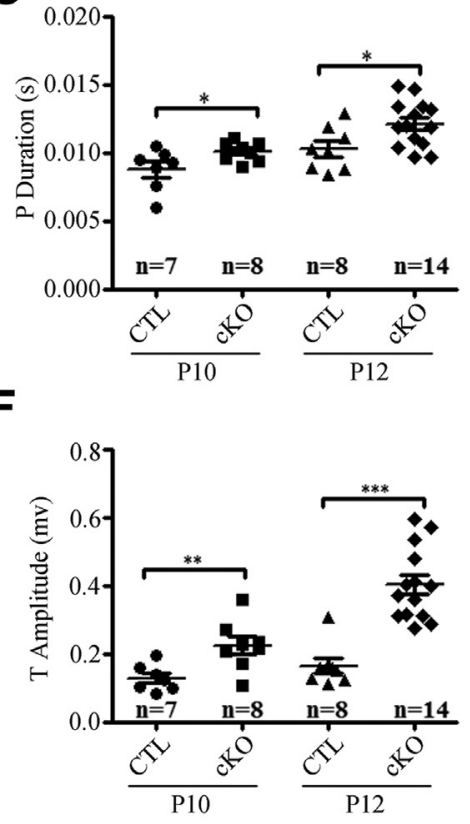

Figure 3 Wdr1 cKO mice exhibit prolonged QT interval and increased T-wave amplitude. A-F: Quantification of heart rate and electrocardiographic parameters. Compared with CTL mice, the heart rate began to decrease at P10 and was further reduced at P12 in cK0 mice (A). There were no differences in PR interval between the two groups (B), but the P duration began to increase at P10 and was further increased at P12 (C). There were no differences in QRS interval between the two groups (D). The QT interval was unchanged at P10, but prolonged at P12 in cK0 mice (E). A significant increase in T-wave amplitude starting at P10 was observed in $\mathrm{CKO}$ mice (F). $\mathbf{G}$ and $\mathbf{H}$ : Representative electrocardiographic recordings at P12 show significantly increased T-wave amplitude in cKO mice. Data are expressed as individual data points and as means \pm SEM. ${ }^{*} P<0.05,{ }^{*} P<0.01$, and ${ }^{* * *} P<0.001$.

At P12, however, cardiomyocytes of cKO mice were larger, compared with CTL, indicating hypertrophic growth of cardiomyocytes (Figure 2, G, K, and L). TUNEL and Ki-67 staining did not reveal any differences between cKO and CTL hearts, indicating that cell proliferation and apoptosis were unchanged in cKO mice (data not shown). Gene expression analysis revealed substantially increased mRNA levels of the two hypertrophic markers ANP and BNP in the cKO heart, compared with CTL, indicating an activated hypertrophic program in cKO mice. However, the mRNA expression levels of $\alpha$-MHC and $\beta$-MHC were unchanged in $\mathrm{cKO}$ mice (Figure 2M).

Echocardiographic study revealed no differences in heart function between $\mathrm{CKO}$ and CTL mice at P10. At P12 and later, however, $\mathrm{EF}$ and $\mathrm{FS}$ were decreased in $\mathrm{cKO}$ mice (Figure 2, $\mathrm{N}$ and $\mathrm{O}$ ). Left ventricular internal diameter in diastole was significantly decreased (Figure 2P) and interventricular septum in diastole was increased (Figure 2Q) in cKO mice. These results indicate that the cKO heart is hypertrophic and that contraction function of the left ventricle is impaired (Table 1).

\section{Wdr1 cK0 Mice Exhibit Prolonged QT Interval and} Increased ST Height and T-Wave Amplitude

To examine the electrical activity of $W d r l$ cKO mice, we performed electrocardiographic analysis. At P10, a significant decrease of heart rate was observed in $W d r l$ cKO mice (417 \pm 11 beats per minute), compared with CTL $(447 \pm 19$ beats per minute) (Figure 3A). At P12, the heart rate was further reduced in $\mathrm{cKO}$ mice ( $383 \pm 39$ beats per minute), compared with CTL $(453 \pm 58$ beats per minute). The PR interval did not differ between $\mathrm{cKO}$ and CTL mice (Figure 3B). Compared with CTL mice, cKO mice exhibited prolonged $\mathrm{P}$ duration starting at $\mathrm{P} 10$ (Figure $3 \mathrm{C}$ ), whereas the QRS waves were similar in the two groups (Figure 3D). No differences were detected in QT interval and ST height between CTL and cKO mice at P10. At P12, however, the QT interval was prolonged (Figure 3E) and the ST height was elevated (data not shown) in cKO mice. Beginning at P10, a significant increase in $\mathrm{T}$-wave amplitude was observed In cKO mice (Figure 3, F-H). Taken together, the abnormalities of QT interval, ST height, and T-wave amplitude in cKO 


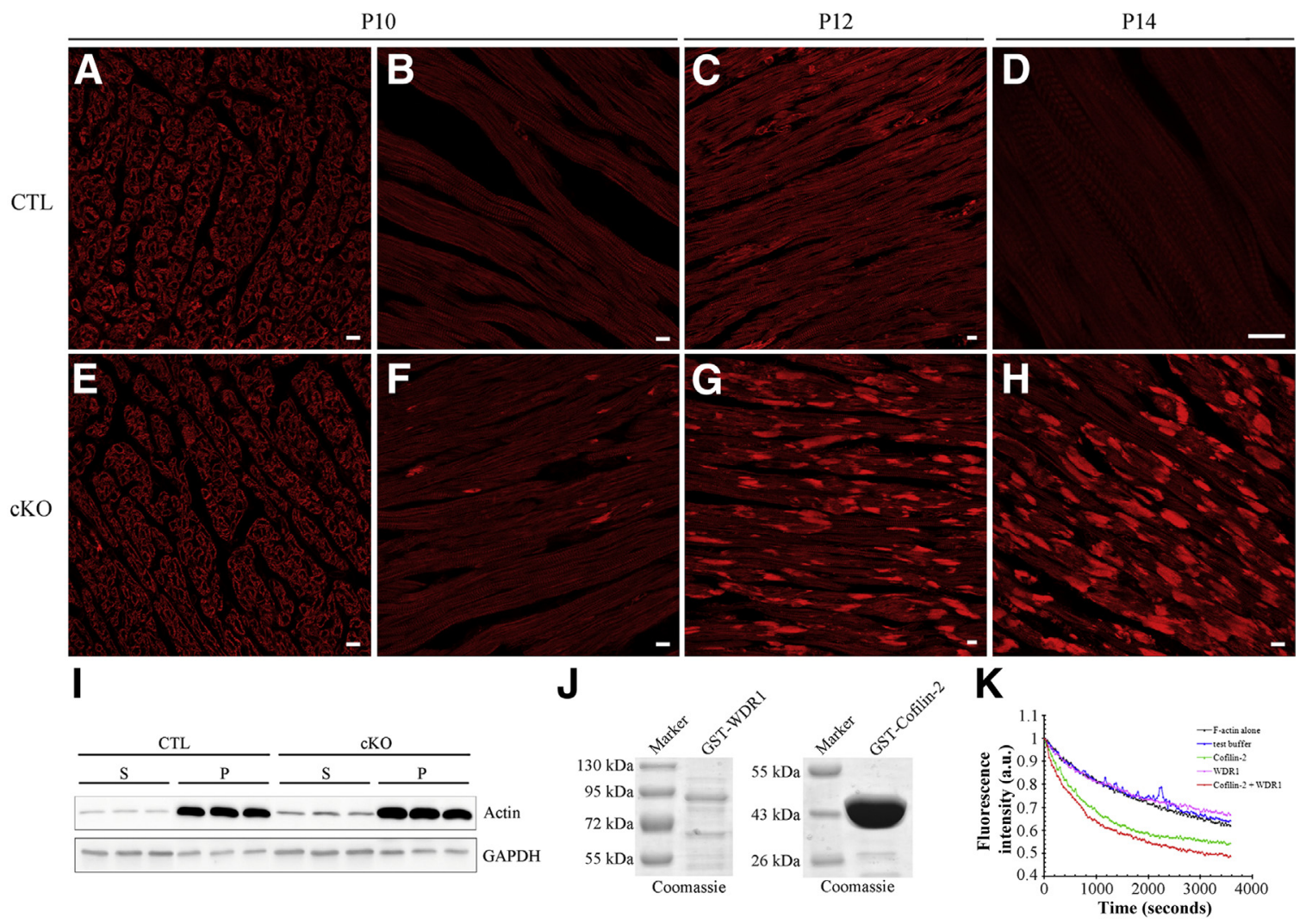

Figure 4 F-actin accumulations in the myocardium of Wdr1 cKO mice. A-H: Immunofluorescence staining for phalloidin. Compared with CTL (A-D), mild F-actin accumulations were observed in $\mathrm{CKO}$ mice at P10 (E and F); large amounts of F-actin accumulations were observed at P12 (G), with further increases at P14 (H). I: Protein extracts from frozen hearts of CTL and cK0 mice were separated into F-actin (pellet) and G-actin (supernatant) fractions by centrifugation for Western blotting analysis using a pan-actin antibody. Both actins were increased in cKO mice, compared with CTL. J: Recombinant GST-WDR1 and GST-cofilin-2 fusion proteins were subjected to SDS-PAGE and stained with Coomassie Brilliant Blue as a test of expression and purification. K: In vitro assay for actin depolymerization kinetics by measuring a reduction of pyrene F-actin fluorescence signal. The depolymerization rate was increased as expected when recombinant GST-cofilin-2 was added, whereas adding recombinant GST-WDR1 alone did not affect the depolymerization rate. Addition of both GST-WDR1 and GST-cofilin-2 caused more significantly accelerated F-actin depolymerization, compared with GST-cofilin-2 alone. Scale bars $=10 \mu \mathrm{m}$.

mice indicate abnormalities of ventricular electrocardiographic repolarization.

\section{F-Actin Accumulations in the Myocardium of Wdr1 cKO Mice}

Cardiac sarcomeric actin filaments have recently been shown to exhibit significant actin dynamics, ${ }^{11,16}$ and AIP1 accelerates ADF/cofilin-mediated actin dynamics. ${ }^{5}$ To determine whether $W d r l$ is required for actin dynamics and turnover in cardiac muscle, we used phalloidin staining of frozen heart sections to specifically label F-actin. At P10, mild F-actin accumulations were found in cKO mice (Figure 4, A, B, E, and $\mathrm{F}$ ). At P12, cKO mice exhibited a large amount of F-actin accumulations (Figure 4, C and G), which became even more dramatic by P14 (Figure 4, D and H). To further determine whether the increase in total actin level is due to increase in the filamentous form of actin (F-actin) or the monomeric form of actin (G-actin) or both, we measured the levels of F-actin and G-actin separately in the heart of cKO and CTL mice. We found significantly increased levels of both F-actin (pellet fraction) and G-actin (supernatant fraction) in $\mathrm{cKO}$ mice at P12 (Figure 4I). Consistent with previous studies, F-actin was the predominant form of actin in both cKO and CTL hearts, making up more than $90 \%$ of the total actin (data not shown).

To determine whether WDR1 regulates the actin depolymerization, we expressed and purified recombinant GSTWDR1 and GST-cofilin-2 (muscle form) fusion proteins (Figure 4J), and then performed an in vitro actin depolymerization assay. In this assay, pyrene-labeled muscle G-actin is initially polymerized into fluorescent F-actin, an unstable structure that is subsequently allowed to depolymerize. The depolymerization process was measured in real time and recorded as a reduction in the pyrene fluorescence signal over time. When GST-cofilin-2 was added to the reaction mixture, the depolymerization rate was strongly increased (as expected), whereas GST-WDR1 alone did not affect the depolymerization rate, in accord with previous report. ${ }^{5}$ Importantly, addition of GST-WDR1 and GST-cofilin-2 together caused significantly more accelerated F-actin depolymerization, compared with adding GST-cofilin-2 alone 

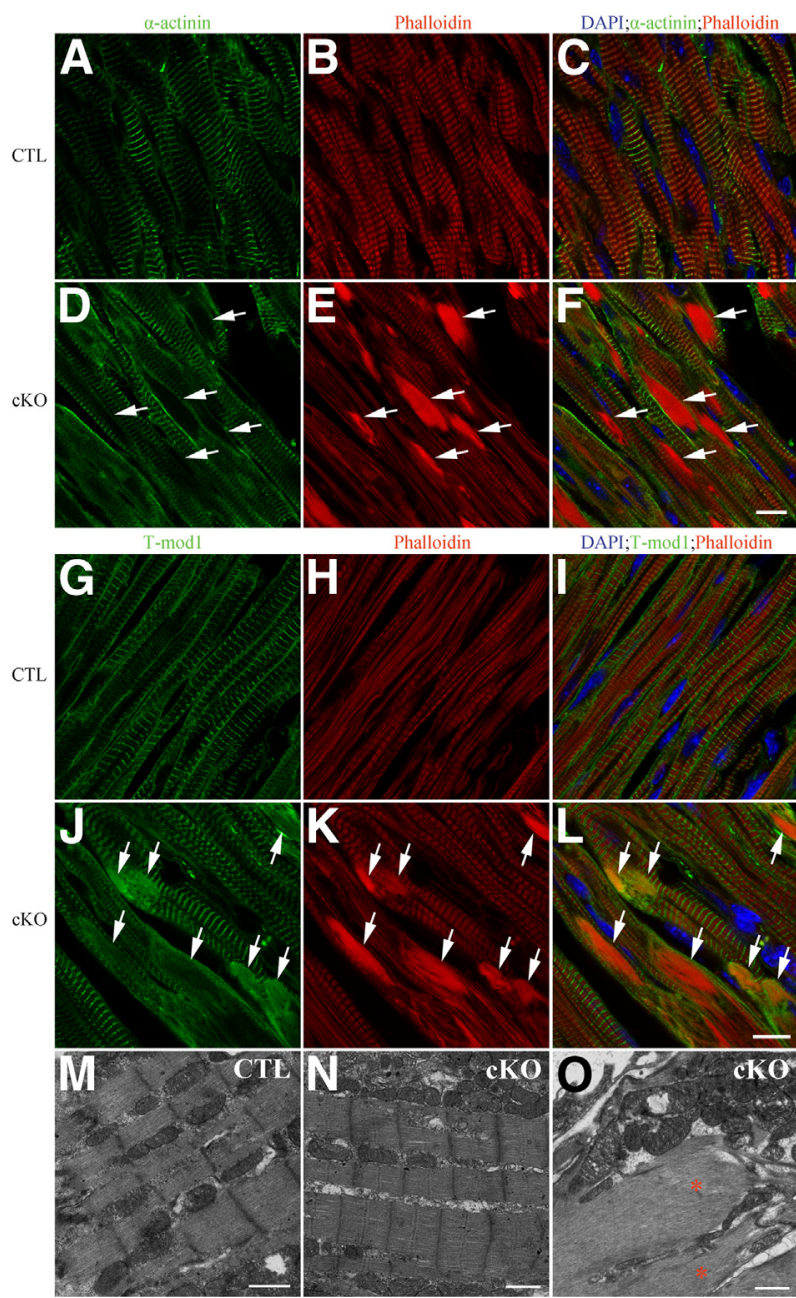

Figure 5 Cardiac muscles in Wdr1 cK0 mice exhibit severe disruptions of sarcomeric structure. A-L: Immunofluorescence microscopy analysis of sarcomeric structures and actin filament accumulations at P12. In CTL myofibrils, $\alpha$-actinin and Tmod1 were aligned in striation $(\mathbf{A}-\mathbf{C}$ and $\mathbf{G}-\mathbf{I})$. In CKO myofibrils, however, the sarcomeric structure was severely disrupted, and the striated pattern was lost in regions where strong $\mathrm{F}$-actin accumulations occur ( $\mathbf{D}-\mathbf{F}$ and $\mathbf{J}-\mathbf{L}$ ). Within the actin aggregates, $\alpha$ actinin staining was strongly reduced (D-F), and Tmod1 staining was diffused but robust in CKO myofibrils ( $\mathbf{J}-\mathbf{L}$ ) (arrows). $\mathbf{M}-\mathbf{0}$ : Transmission EM analysis of hearts at P12. Sarcomeres from CTL and most areas of CKO cardiac muscles had a normal looking structure, with distinct $A$ bands and narrow and uniformly spaced $\mathbf{Z}$ lines ( $\mathbf{M}$ and $\mathbf{N}$ ). However, in areas of myofibrillar disruption in cKO muscle $(\mathbf{0})$, strong F-actin accumulations were present (asterisks). No nemaline bodies were detected in CKO myofibrils. Scale bars: $10 \mu \mathrm{m}(\mathbf{A}-\mathbf{L}) ; 1 \mu \mathrm{m}(\mathbf{M}-\mathbf{0})$.

(Figure 4K). These results indicate that WDR1 accelerates cofilin-mediated actin depolymerization.

\section{Cardiac Muscles in Wdr1 cK0 Mice Exhibit Severe Disruptions of Sarcomeric Structure}

To examine the sarcomeric structure in the myocardium of $W d r l$ cKO mice, we performed double immunostaining for F-actin and the sarcomeric structural component $\alpha$-actinin or the pointed end capper Tmod1 on frozen sections of heart samples, using antibodies of sarcomeric $\alpha$-actinin $(\alpha$-actinin-2 and $\alpha$-actinin- 3 ) and Tmod1, respectively. $W d r l$ cKO heart at P10 exhibited mild F-actin accumulations, and the myofibrillar components $\alpha$-actinin and Tmod 1 appeared mostly undisturbed (data not shown). At P12, in CTL myofibrils and in cKO myofibrils without F-actin aggregates, both $\alpha$-actinin and Tmod 1 were aligned in striation (Figure 5, A-L). In regions of strong F-actin accumulation in cKO myofibrils, however, the sarcomeric structure was severely disrupted and the striated pattern was lost (Figure 5, D-F and $\mathrm{J}-\mathrm{L}$ ). Within actin aggregates, sarcomeric $\alpha$-actinin staining was strongly reduced (Figure 5, D-F) and Tmod1 staining was diffused but robust (Figure 5, J-L), in contrast to the striated distribution pattern in CTL myofibrils. These staining patterns suggest that the strongly accumulated actin filaments still have Tmod 1 capping their pointed ends, even though the overall arrangement of filaments is in disarray, whereas the Z-line structure labeled by the sarcomeric $\alpha$-actinin staining was almost totally dissolved.

To determine the structure of WDR1-deficient cardiac muscle in greater detail, transmission electron microscopy was performed on cKO and CTL samples. At P12, sarcomeres from CTL samples and most areas of cKO (P12) cardiac muscle had a normal looking structure, with distinct $\mathrm{A}$ bands and narrow and uniformly spaced $\mathrm{Z}$ lines (Figure 5, $\mathrm{M}$ and $\mathrm{N}$ ). However, strong $\mathrm{F}$-actin accumulations were present in areas of myofibrillar disruptions in cKO muscle, (Figure 5O). We did not detect apparent nemaline bodies in cKO cardiac muscles from the transmission electron microscopy data, which was consistent with Gömöri trichrome staining results performed on frozen sections of CTL and cKO heart (data not shown).

Increased Levels of Sarcomeric Proteins and Cofilins in the Heart of Wdr1 cKO Mice

Western blotting of hearts from cKO and CTL mice was performed at P12 to evaluate several sarcomeric proteins. Expression levels of the F-actin binding proteins tropomyosin, cardiac troponin $\mathrm{T}(\mathrm{cTnT})$, and cardiac troponin $\mathrm{I}$ (cTnI) were significantly elevated in cKO heart, compared with CTL. Expression of the F-actin pointed end capping protein Tmod1 was also increased, whereas expression of the Z-band protein sarcomeric $\alpha$-actinin was unchanged (Figure 6, A-C). The increase in levels of these F-actin binding or capping proteins likely reflects the overall accumulations of F-actin in cKO cardiomyocytes. Western blot analysis revealed a large increase of total actin level at P12 (Figure 6, A and C). At P10, however, no significant increase in total actin was detected (data not shown). Five of the six actin isoforms present in cardiac muscle were investigated by real-time qPCR analysis, using samples of heart from Wdrl cKO and CTL mice at P10. mRNA expression levels of $\alpha$-skeletal muscle actin and aortic smooth muscle actin were significantly increased and those of $\alpha$-cardiac muscle actin-1 were decreased (Figure 6E). 

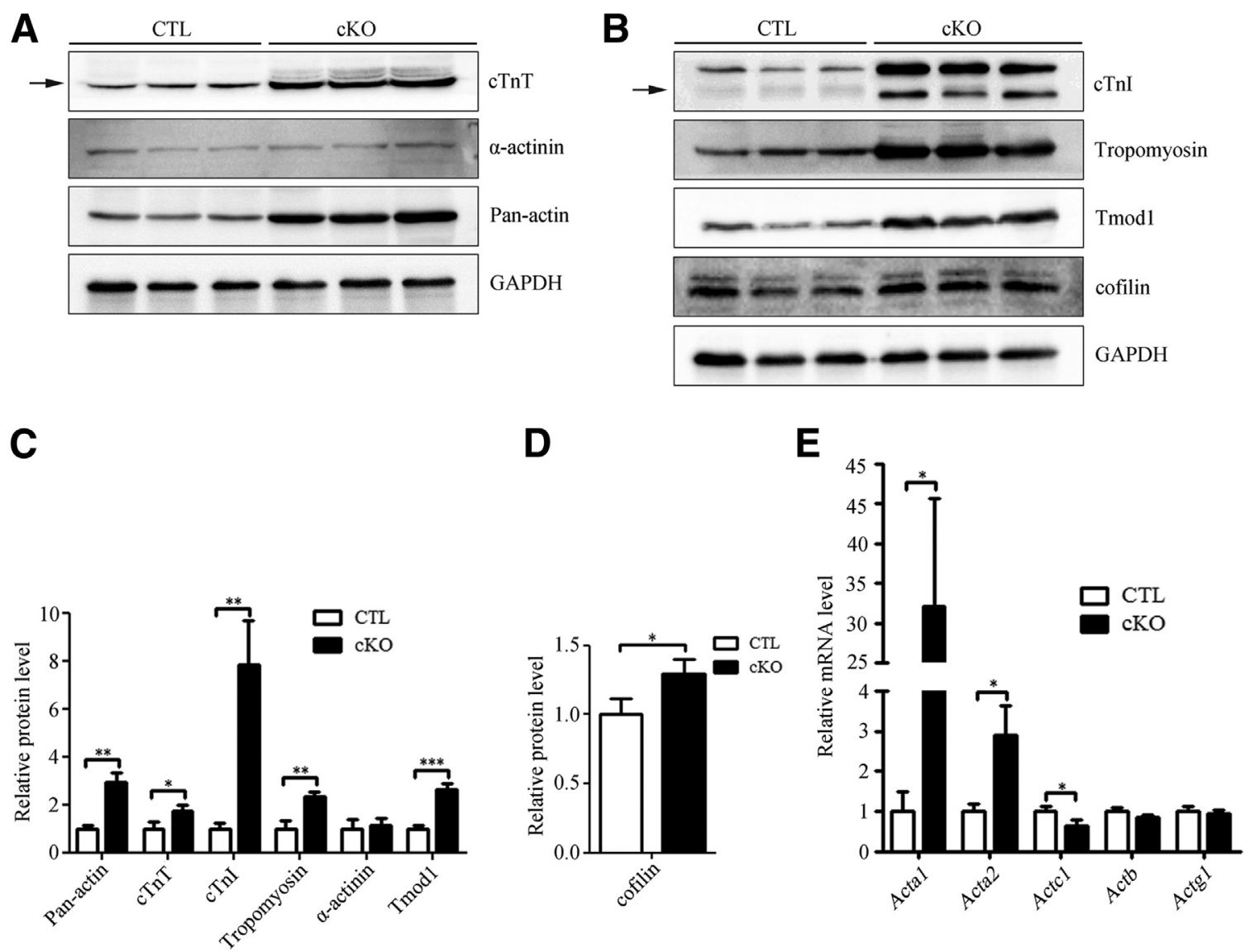

Figure 6 Increased levels of sarcomeric proteins and total cofilin in Wdr1 CKO mouse heart. A-D: Western blotting analysis (A and B) and quantification ( $(\mathbf{a n d} \mathbf{D})$ at P12 revealed increased levels of Tmod1, cTnT, cTnI, tropomyosin, pan-actin, and total cofilin in the cK0 heart, but not of sarcomeric $\alpha$-actinin. The target protein band is indicated by an arrow. E: mRNA expression levels of five actin isoforms in the heart at P10. Acta1 and Acta2 were increased and Actc1 was decreased in CKO mice, compared with CTL; Actb and Actg1 were unchanged. Data are expressed as means \pm SEM. $n=3$ mice per group. ${ }^{*} P<0.05$, $* * P<0.01$, and $\left.{ }^{* * *} P<0.001\right)$.

Using an antibody detecting both isoforms of cofilin, we found significantly increased protein level of total cofilin (ie, both cofilin-1 and cofilin-2) in Wdrl cKO heart (Figure 6, B and $\mathrm{D}$ ), and the increased total cofilin could be detected as early as P10 (data not shown). To determine whether there was any alteration in cofilin localization in cardiac muscle, immunofluorescence was performed on CTL and cKO samples at P12. Cofilins exhibited a diffuse and uniform distribution pattern in CTL myofibrils and in regions of cKO myofibrils without actin aggregates (Figure 7, A-L); by contrast, cofilin staining strongly colocalized with F-actin accumulations in cKO myofibrils (Figure 7, D-F and $\mathrm{J}-\mathrm{L}$ ).

\section{Deleting Wdr1 in Adult Mouse Heart Also Results in Heart Hypertrophy, F-Actin Accumulation within Myofibrils, and Lethality}

To determine whether AIP1 plays essential roles in maintenance of adult cardiomyocytes, adult cardiomyocyte-specific $W d r l$ deletion was performed using tamoxifen-induced aMHC-MerCreMer mice. ${ }^{32}$ Crossing $W d r l^{f / f}$ mice with aMHC-MerCreMer mice generated mice allowing tamoxifeninduced specific deletion of $W d r l$ in cardiomyocytes. At 2 months of age, $W d r l^{f f f} ; \alpha \mathrm{MHC}-\mathrm{MerCreMer}$ and $W d r l^{f f f}$ mice were treated with tamoxifen by intraperitoneal injection, resulting in $W d r l$ iKO mice. $W d r l$ excision was confirmed by Western blotting at 10 days after the first tamoxifen injection (Figure 8A). Most iKO mice started dying nearly 2 months after the first tamoxifen injection, and none survived past 104 days (Figure 8B). Body weight did not differ between iKO mice and CTL littermates (data not shown). The heart weight/body weight ratio began to increase in male iKO mice at 1 month after tamoxifen injection (Figure 8C), but no significant changes were observed in female iKO mice (data not shown).

The iKO mice exhibited increased cross-sectional surface area (Figure 8D) and F-actin accumulation (Figure 8E) at 1 month after the first tamoxifen injection. Taken together, these results indicate that AIP1-regulated actin dynamics are essential in cardiac muscle of both infant and adult mice.

\section{Discussion}

With the present study, we have demonstrated that AIP1 is required for cardiomyocyte growth at the postnatal stage. 

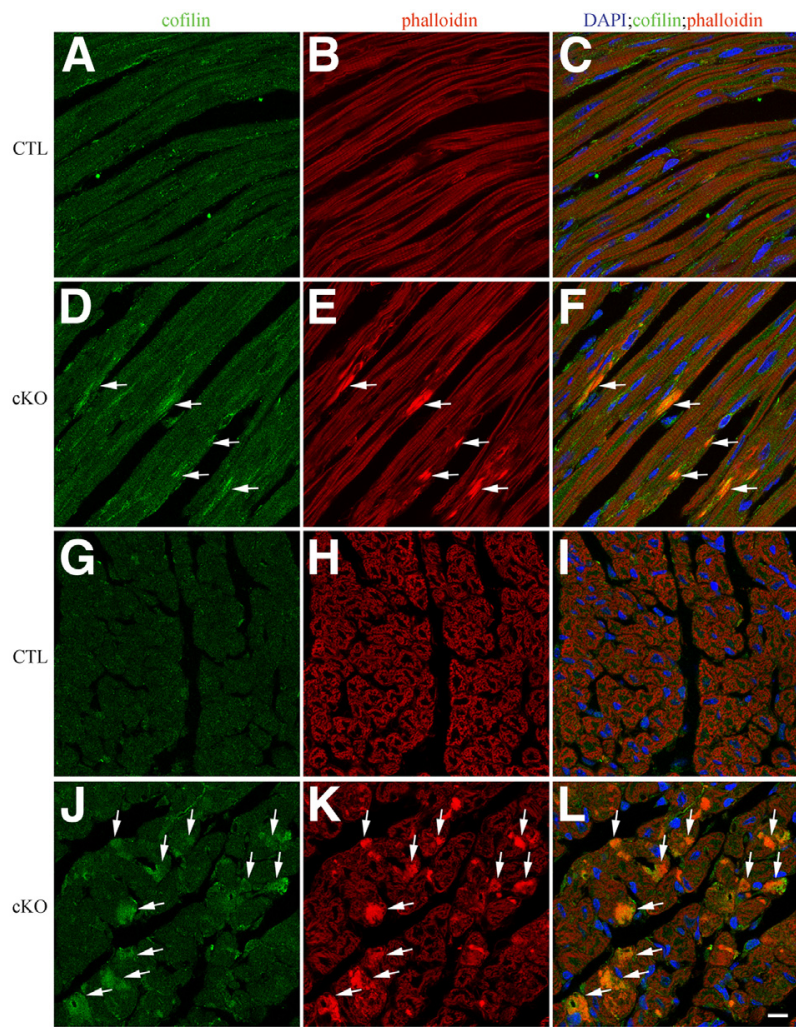

Figure 7 Total cofilin is enriched in the accumulated filament actin of Wdr1 cK0 mice. Immunofluorescence staining at P12 of longitudinal sections $(\mathbf{A}-\mathbf{F})$ and cross sections $(\mathbf{G}-\mathbf{L})$. Total cofilin was abundant in myofibrils where $\mathbf{F}$-actin accumulations were present (arrows) (D-F and $\mathbf{J}-\mathbf{L})$. Scale bar $=10 \mu \mathrm{m}$.

Before birth, mammalian embryonic heart growth is achieved mainly by cardiomyocyte division, and the myofibrils undergo a disassembly-reassembly cycle during cell division in cultured cardiomyocytes. ${ }^{33}$ Shortly after birth, cardiomyocytes lose the ability to divide, and postnatal heart growth is due to increase of cardiomyocyte volume by addition of sarcomeric actin filaments at the sides and tips of growing myotubes. ${ }^{7} \mathrm{At}$ adulthood, however, myocardium ceases growth as myofibrils become mature and the actin dynamics regulators at the pointed ends maintain constant length of sarcomeric actin filaments. ${ }^{34}$ Our present data suggest that the actin severing and disassembly function of AIP1 is required for efficient actin filament addition during postnatal myofibril growth, because $W d r l$ deletion results in sarcomeric disruption at P12. This is consistent with previous findings that loss of function of mammalian $\mathrm{ADF} / \mathrm{cofilins}$ both in vivo and in vitro disrupts sarcomeric structure in striated muscles, suggesting that AIP1 promotes cofilin-mediated actin dynamics during postnatal cardiomyocyte growth.

From P5 to P14, the murine heart undergoes dramatic hypertrophic growth to meet the increased demands of blood output. ${ }^{35}$ This could be the reason why most of the phenotypes started to appear after P10 in our cKO mice. Notably, these mice underwent sudden death, beginning at P13. Sarcomere assembly was likely defective in these mice, as
A

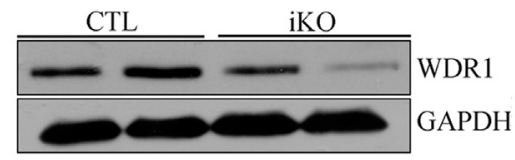

B

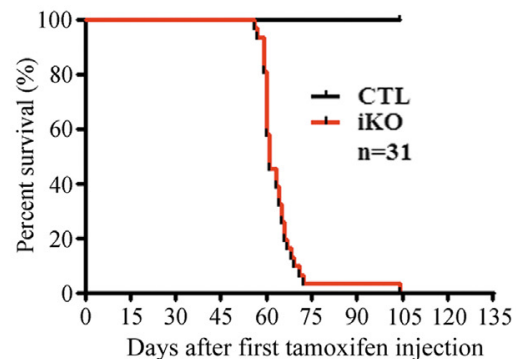

C

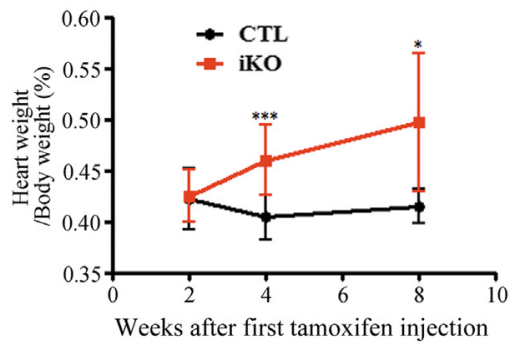

D
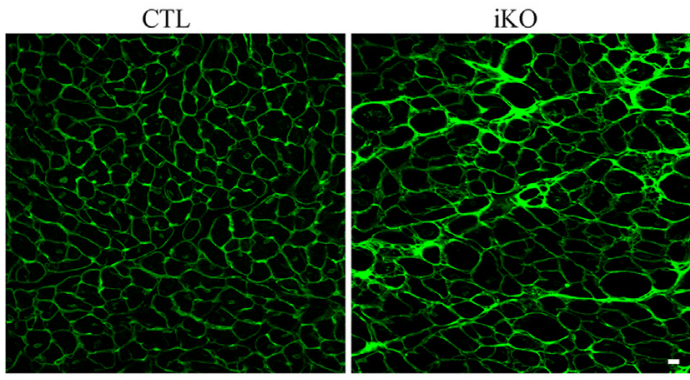

E
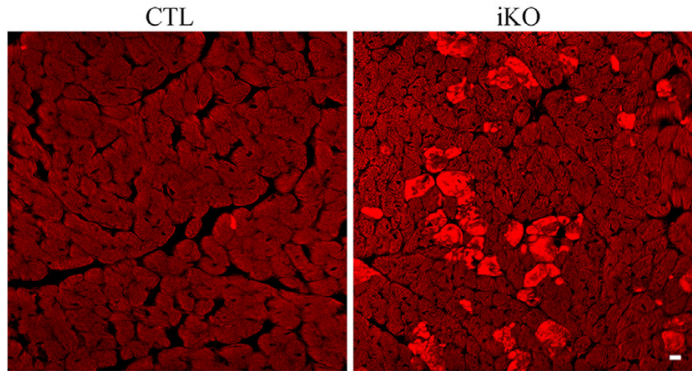

Figure 8 Wdr1 deletion in adult mouse heart results in heart hypertrophy, F-actin accumulations within myofibrils, and lethality. A: At 10 days after tamoxifen administration, hearts were dissected for Western blotting to detect deletion efficiency. WDR1 protein levels were substantially decreased in iKO mice, compared with CTL. B: The iKO mice began to die at 56 days, and none survived past 104 days after tamoxifen administration. C: The heart weight/body weight ratio began to increase in male iKO mice at 1 month after tamoxifen administration, but there was no significant change in CTL mice. D and E: Heart cross sections at 1 month after tamoxifen administration. In iKO mice, cardiomyocyte size (D, wheat germ agglutinin stain) was increased, compared with CTL, and F-actin accumulations (E, phalloidin staining) compared with $\mathrm{CTL}$. ${ }^{*} P<0.05$; $* * * P<0.001$. Scale bar $=10 \mu \mathrm{m}$. 
indicated by disrupted sarcomeric structure, which may lead to severe defects in heart contractility and thus to sudden death. Our results suggest that WDR1 plays critical roles in actin dynamics regulation for sarcomere assembly during this dramatic phase of cardiomyocyte hypertrophic growth.

The strong actin accumulations in Wdrl-deleted cardiomyocytes further suggest that AIP1 likely enhances the roles of cofilin- 1 and cofilin-2 in disassembling or removing an excess of nonproductive actin filaments in cardiomyocytes, as was proposed in a recent report to be essential for sarcomere maturation. ${ }^{16}$ Interestingly, in another recent study with Ckmm-Cre deletion of the cofilin-2 gene (Cfl2) in the postnatal heart in mice, the authors did find strong disruptions in sarcomeres or actin accumulations, ${ }^{17}$ which might be due to compensation effect by the undeleted cofilin-1. The genetic evidence that $W d r l$ deletion caused an apparently more severe phenotype in the postnatal heart, compared with $C f l 2$ deletion, implies that AIP1 works with both cofilin-1 and cofilin-2 and that removing AIP1 function causes reduction of activity for both cofilins.

Interestingly, $W d r l$ deletion results in an alteration of the distribution pattern of total cofilin, along with a significant increase of their overall protein levels. In Wdrl-deleted cardiomyocytes, total cofilin becomes strongly enriched in areas of actin accumulation in sarcomeres, whereas it is uniformly distributed throughout the cytoplasm of CTL cardiomyocytes. This phenotype is consistent with observations in nematode body wall muscle and Drosophila eye epithelial cells, in which AIP1 loss of function resulted in cofilin being highly enriched in ectopic F-actin-accumulated structures in these two cell types. $^{6,22}$ Our present results suggest that there might be a conserved mechanism that senses the presence of accumulated F-actin, elevates expression of cofilin, and then strongly localizes cofilin to those ectopic structures, attempting to disassemble them. Alternatively, a previous study suggests that ectopic cofilin enrichment reflects the failure of disassembly of cofilin-bound actin filaments and thus the accumulation of cofilin. ${ }^{22}$ However, this reasoning cannot fully explain the overall increase of cofilin protein levels.

Furthermore, cardiomyocyte-specific Wdrl deletion causes strong functional defects in the mouse heart, leading to postnatal sudden death (beginning at P13). At P12, the cKO mice exhibited significant heart hypertrophy and impaired contraction function of left ventricle, as revealed by echocardiography data. The phenotype of cardiac hypertrophy was not surprising, because the mutations causing hypertrophic cardiomyopathy have all been identified in genes encoding many of the sarcomeric proteins, including $\beta$-myosin heavy chain, $\alpha$-tropomyosin, troponin I, troponin $\mathrm{T}$, and actin. ${ }^{36}$ Notably, the hypertrophic hearts of cKO mice did not exhibit the $\alpha$ MHC-to- $\beta \mathrm{MHC}$ isoform switch previously reported in many cases of cardiac hypertrophy. However, some researchers have proposed that the isoform switch is not obligatorily coupled with hypertrophy, ${ }^{37}$ although the underlying mechanism is not known. Thus, this phenotype does not resemble canonical cardiac hypertrophy. It is likely that the disruption of actin filament assembly in the absence of $W d r l$ initiates a secondary heart remodeling, which could be distinct from canonical cardiac hypertrophy. Hypertrophy is reported to be a risk factor for QT prolongation and cardiac sudden death. ${ }^{38}$ Electrocardiographic recordings revealed decreased heart rate and prolonged QT interval.

In humans, recessive cofilin- 2 mutants had been associated with nemaline myopathy. ${ }^{18,19}$ Cfl2-deleted mice also exhibited nemaline-like bodies, along with F-actin accumulations, sarcomeric disruptions, and muscle weakness in the skeletal muscles. ${ }^{17}$ Our transmission electron microscopy analysis and Gömöri trichrome staining did not reveal significant nemaline-like bodies; however, a previous report suggests that, although effective in skeletal muscle, Gömöri trichrome staining may not be efficient in detecting nemaline bodies in myocardium tissues. ${ }^{39,40}$ Thus, the role of AIP1 in nemaline myopathy remains to be determined. Furthermore, the F-actin aggregates in cKO myofibrils did not contain $\alpha$-actinin, whereas nemaline bodies are usually $\alpha$-actinin positive. ${ }^{41}$ Taken together, these results suggest that AIP1 deficiency causes sarcomeric disruptions during postnatal myocardium growth, which likely leads to impaired contraction and heart hypertrophy, as well as disruption of electrical heart function, resulting in sudden cardiac death in cKO mice.

Finally, our findings that $W d r l$ deletion in the adult mouse heart results in heart hypertrophy, F-actin accumulations within myofibrils, and lethality demonstrate that AIP1 is also required for cardiomyocyte maintenance at the adult stage. This result suggests that mature cardiomyocytes also require a certain level of actin disassembly and turnover to regulate their maintenance function. However, in adult heart the level of actin dynamics needed is likely significantly reduced, compared with that in the postnatal heart, because the iKO mice exhibited milder defects in the heart and less sudden lethality than the cKO mice. Consistently, the expression of AIP1 in the heart decreased gradually after birth, eventually reaching a low level at adulthood. This suggests that AIP1 and its function in promoting actin dynamics are more critical during postnatal cardiac muscle growth than in maintenance of adult muscle.

\section{Acknowledgments}

We thank Dr. Xiao Yang in Beijing for providing the aMHC-Cre mice and Wendong Cheng for maintaining the Wdrl mouse colony.

\section{References}

1. Cooper JA, Schafer DA: Control of actin assembly and disassembly at filament ends. Curr Opin Cell Biol 2000, 12:97-103

2. Machesky LM, Gould KL: The Arp2/3 complex: a multifunctional actin organizer. Curr Opin Cell Biol 1999, 11:117-121

3. Pruyne D, Evangelista M, Yang C, Bi E, Zigmond S, Bretscher A, Boone C: Role of formins in actin assembly: nucleation and barbedend association. Science 2002, 297:612-615

4. Bamburg JR: Proteins of the ADF/cofilin family: essential regulators of actin dynamics. Annu Rev Cell Dev Biol 1999, 15:185-230 
5. Ono S: Regulation of actin filament dynamics by actin depolymerizing factor/cofilin and actin-interacting protein 1: new blades for twisted filaments. Biochemistry 2003, 42:13363-13370

6. Chu D, Pan H, Wan P, Wu J, Luo J, Zhu H, Chen J: AIP1 acts with cofilin to control actin dynamics during epithelial morphogenesis. Development 2012, 139:3561-3571

7. Ono S: Dynamic regulation of sarcomeric actin filaments in striated muscle. Cytoskeleton (Hoboken) 2010, 67:677-692

8. Gregorio CC, Weber A, Bondad M, Pennise CR, Fowler VM: Requirement of pointed-end capping by tropomodulin to maintain actin filament length in embryonic chick cardiac myocytes. Nature 1995, 377: $83-86$

9. Schafer DA, Hug C, Cooper JA: Inhibition of CapZ during myofibrillogenesis alters assembly of actin filaments. J Cell Biol 1995, 128 : $61-70$

10. Dome JS, Mittal B, Pochapin MB, Sanger JM, Sanger JW: Incorporation of fluorescently labeled actin and tropomyosin into muscle cells. Cell Differ 1988, 23:37-52

11. Imanaka-Yoshida K, Sanger JM, Sanger JW: Contractile protein dynamics of myofibrils in paired adult rat cardiomyocytes. Cell Motil Cytoskeleton 1993, 26:301-312

12. Shimada Y, Suzuki H, Konno A: Dynamics of actin in cardiac myofibrils and fibroblast stress fibers. Cell Struct Funct 1997, 22:59-64

13. Suzuki H, Komiyama M, Konno A, Shimada Y: Exchangeability of actin in cardiac myocytes and fibroblasts as determined by fluorescence photobleaching recovery. Tissue Cell 1998, 30:274-280

14. Littlefield R, Almenar-Queralt A, Fowler VM: Actin dynamics at pointed ends regulates thin filament length in striated muscle. Nat Cell Biol 2001, 3:544-551

15. Wang J, Shaner N, Mittal B, Zhou Q, Chen J, Sanger JM, Sanger JW: Dynamics of Z-band based proteins in developing skeletal muscle cells. Cell Motil Cytoskeleton 2005, 61:34-48

16. Skwarek-Maruszewska A, Hotulainen P, Mattila PK, Lappalainen P: Contractility-dependent actin dynamics in cardiomyocyte sarcomeres. J Cell Sci 2009, 122:2119-2126

17. Agrawal PB, Joshi M, Savic T, Chen Z, Beggs AH: Normal myofibrillar development followed by progressive sarcomeric disruption with actin accumulations in a mouse $\mathrm{Cfl} 2$ knockout demonstrates requirement of cofilin-2 for muscle maintenance. Hum Mol Genet 2012, 21:2341-2356

18. Agrawal PB, Greenleaf RS, Tomczak KK, Lehtokari VL, WallgrenPettersson C, Wallefeld W, Laing NG, Darras BT, Maciver SK, Dormitzer PR, Beggs AH: Nemaline myopathy with minicores caused by mutation of the CFL2 gene encoding the skeletal muscle actinbinding protein, cofilin-2. Am J Hum Genet 2007, 80:162-167

19. Ockeloen CW, Gilhuis HJ, Pfundt R, Kamsteeg EJ, Agrawal PB, Beggs AH, Dara Hama-Amin A, Diekstra A, Knoers NV, Lammens M, van Alfen N: Congenital myopathy caused by a novel missense mutation in the CFL2 gene. Neuromuscul Disord 2012, 22: 632-639

20. Clarkson E, Costa CF, Machesky LM: Congenital myopathies: diseases of the actin cytoskeleton. J Pathol 2004, 204:407-417

21. Costa CF, Rommelaere H, Waterschoot D, Sethi KK, Nowak KJ, Laing NG, Ampe C, Machesky LM: Myopathy mutations in alphaskeletal-muscle actin cause a range of molecular defects. J Cell Sci 2004, 117:3367-3377

22. Ono S: The Caenorhabditis elegans unc-78 gene encodes a homologue of actin-interacting protein 1 required for organized assembly of muscle actin filaments [Erratum appeared in J Cell Biol 2001, 153: 888]. J Cell Biol 2001, 152:1313-1319

23. Mohri K, Ono S: Actin filament disassembling activity of Caenorhabditis elegans actin-interacting protein 1 (UNC-78) is dependent on filament binding by a specific ADF/cofilin isoform. J Cell Sci 2003, 116:4107-4118
24. Mohri K, Ono K, Yu R, Yamashiro S, Ono S: Enhancement of actindepolymerizing factor/cofilin-dependent actin disassembly by actininteracting protein 1 is required for organized actin filament assembly in the Caenorhabditis elegans body wall muscle. Mol Biol Cell 2006, 17 : 2190-2199

25. Yamashiro S, Cox EA, Baillie DL, Hardin JD, Ono S: Sarcomeric actin organization is synergistically promoted by tropomodulin, ADF/cofilin, AIP1 and profilin in C. elegans. J Cell Sci 2008, 121: 3867-3877

26. Ono S, Nomura K, Hitosugi S, Tu DK, Lee JA, Baillie DL, Ono K: The two actin-interacting protein 1 genes have overlapping and essential function for embryonic development in Caenorhabditis elegans. Mol Biol Cell 2011, 22:2258-2269

27. Kile BT, Panopoulos AD, Stirzaker RA, Hacking DF, Tahtamouni LH, Willson TA, Mielke LA, Henley KJ, Zhang JG, Wicks IP, Stevenson WS, Nurden P, Watowich SS, Justice MJ: Mutations in the cofilin partner Aip1/Wdr1 cause autoinflammatory disease and macrothrombocytopenia. Blood 2007, 110:2371-2380

28. Farley FW, Soriano P, Steffen LS, Dymecki SM: Widespread recombinase expression using FLPeR (flipper) mice. Genesis 2000, 28:106-110

29. Zhang QJ, Chen HZ, Wang L, Liu DP, Hill JA, Liu ZP: The histone trimethyllysine demethylase JMJD2A promotes cardiac hypertrophy in response to hypertrophic stimuli in mice. J Clin Invest 2011, 121: $2447-2456$

30. Qi X, Yang G, Yang L, Lan Y, Weng T, Wang J, Wu Z, Xu J, Gao X, Yang X: Essential role of Smad4 in maintaining cardiomyocyte proliferation during murine embryonic heart development. Dev Biol 2007, 311:136-146

31. Wang J, Xu N, Feng X, Hou N, Zhang J, Cheng X, Chen Y, Zhang Y, Yang X: Targeted disruption of Smad4 in cardiomyocytes results in cardiac hypertrophy and heart failure. Circ Res 2005, 97:821-828

32. Sohal DS, Nghiem M, Crackower MA, Witt SA, Kimball TR, Tymitz KM, Penninger JM, Molkentin JD: Temporally regulated and tissue-specific gene manipulations in the adult and embryonic heart using a tamoxifen-inducible Cre protein. Circ Res 2001, 89: $20-25$

33. Ahuja P: Sequential myofibrillar breakdown accompanies mitotic division of mammalian cardiomyocytes. J Cell Sci 2004, 117: 3295-3306

34. Littlefield R, Fowler V: Thin filament length regulation in striated muscle sarcomeres: pointed-end dynamics go beyond a nebulin ruler. Semin Cell Dev Biol 2008, 19:511-519

35. Leu M, Ehler E, Perriard JC: Characterisation of postnatal growth of the murine heart. Anat Embryol (Berl) 2001, 204:217-224

36. Bowles NE, Bowles KR, Towbin JA: The "final common pathway" hypothesis and inherited cardiovascular disease. The role of cytoskeletal proteins in dilated cardiomyopathy. Herz 2000, 25: $168-175$

37. Schultz Jel J, Witt SA, Glascock BJ, Nieman ML, Reiser PJ, Nix SL, Kimball TR, Doetschman T: TGF-beta1 mediates the hypertrophic cardiomyocyte growth induced by angiotensin II. J Clin Invest 2002, 109:787-796

38. Kang YJ: Cardiac hypertrophy: a risk factor for QT-prolongation and cardiac sudden death. Toxicol Pathol 2006, 34:58-66

39. Ishibashi-Ueda H, Imakita M, Yutani C, Takahashi S, Yazawa K, Kamiya T, Nonaka I: Congenital nemaline myopathy with dilated cardiomyopathy: an autopsy study. Hum Pathol 1990, 21:77-82

40. Bergmann M, Kamarampaka M, Kuchelmeister K, Klein H, Koch H: Nemaline myopathy: two autopsy reports. Childs Nerv Syst 1995, 11: 610-615

41. North KN, Laing NG, Wallgren-Pettersson C: Nemaline myopathy: current concepts. The ENMC International Consortium and Nemaline Myopathy [Erratum appeared in J Med Genet 1997, 34:879]. J Med Genet 1997, 34:705-713 